\title{
Architectural Geometry
}

\author{
Helmut Pottmann ${ }^{\mathrm{a}, \mathrm{b}}$, Michael Eigensatz ${ }^{\mathrm{c}}$, Amir Vaxman $^{\mathrm{b}}$, Johannes Wallner ${ }^{\mathrm{d}}$ \\ ${ }^{a}$ King Abdullah University of Science and Technology, Thuwal 23955, Saudi Arabia \\ ${ }^{b}$ Vienna University of Technology, 1040 Vienna, Austria \\ ${ }^{c}$ Evolute GmbH, 1040 Vienna, Austria \\ ${ }^{d}$ Graz University of Technology, 8010 Graz, Austria
}

\begin{abstract}
Around 2005 it became apparent in the geometry processing community that freeform architecture contains many problems of a geometric nature to be solved, and many opportunities for optimization which however require geometric understanding. This area of research, which has been called architectural geometry, meanwhile contains a great wealth of individual contributions which are relevant in various fields. For mathematicians, the relation to discrete differential geometry is significant, in particular the integrable system viewpoint. Besides, new application contexts have become available for quite some old-established concepts. Regarding graphics and geometry processing, architectural geometry yields interesting new questions but also new objects, e.g. replacing meshes by other combinatorial arrangements. Numerical optimization plays a major role but in itself would be powerless without geometric understanding. Summing up, architectural geometry has become a rewarding field of study. We here survey the main directions which have been pursued, we show real projects where geometric considerations have played a role, and we outline open problems which we think are significant for the future development of both theory and practice of architectural geometry.
\end{abstract}

Keywords: Discrete differential geometry, architectural geometry, fabrication-aware design, paneling, double-curved surfaces, single-curved surfaces, support structures, polyhedral patterns, statics-aware design, shading and lighting, interactive modeling

\section{Introduction}

Free forms constitute one of the major trends within contemporary architecture. In its earlier days a particularly important figure was Frank Gehry, with his design approach based on digital reconstruction of physical models, resulting in shapes which are not too far away from developable surfaces and thus ideally suited for his preferred characteristic metal cladding [94]. Nowadays we see an increasing number of landmark buildings involving geometrically complex freeform skins and structures (Fig. 1).

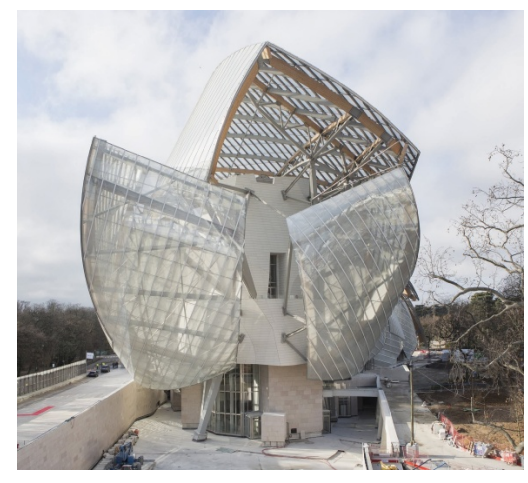

Figure 1: Complex architecture entails a complex workflow. This image shows part of the Fondation Louis Vuitton, Paris, designed by $\mathrm{F}$. Gehry. Photo: Mairie de Paris.

While the modelling of freeform geometry with current tools is well understood, the actual fabrication on the architectural

Email address: helmut .pottmann@kaust.edu.sa (Helmut Pottmann ) scale is a challenge. One has to decompose the skins into manufacturable panels, provide appropriate support structures, meet structural constraints and last, but not least make sure that the cost does not become excessive. Many of these practically highly important problems are actually of a geometric nature and thus the architectural application attracted the attention of the geometric modeling and geometry processing community. This research area is now called Architectural Geometry. It is the purpose of the present survey to provide an overview of this field from the Computer Graphics perspective. We are not addressing here the many beautiful designs which have been realized by engineers with a clever way of using state of the art software, but we are focusing on research contributions which go well beyond the use of standard tools. This research direction has also been inspired by the work of the smart geometry group (www.smartgeometry.com), which promoted the use of parametric design and scripting for mastering geometric complexity in architecture.

From a methodology perspective, it turned out that the probably two most important ingredients for the solution of Architectural Geometry problems are Discrete Differential Geometry (DDG) $[84,16]$ and Numerical Optimization. In order to keep this survey well within Graphics, we will be rather short in discussing the subject from the DDG perspective and only mention those insights which are essential for a successful implementation. It is a fact that understanding a problem from the DDG viewpoint is often equivalent to understanding how to successfully initialize and solve the numerical optimization problems 
which are more directly related to the questions at hand.

In general, the approximation of an ideal design surface by a surface which is suitable for fabrication, is called rationalization in Architecture. This often means panelization, i.e., finding a collection of smaller elements covering the design surface, but it can also mean replacing the design surface by a surface which has a simple generation like a ruled surface. Often, rationalization is harder than the 3D modeling of a surface. A digital modeling tool which automatically generates only buildable structures of a certain type (fabrication-aware design) is probably more efficient than the still prevalent approach based on rationalization. For research, both rationalization and fabricationaware design are interesting, but the latter poses more unsolved problems, at the same time going far beyond architecture.

The solution of the above-mentioned problems may become easier if the shape under consideration has special properties, in which case we do not call it truly freeform. E.g. a surface generated by translation is easily rationalized into flat quadrilaterals (see Fig. 6). Special shapes have been extensively and very successfully employed, but this paper focuses on properties and algorithms relating to arbitrary ("freeform") shapes.

Remark. The reader is advised that we use the word design in its purely technical sense, meaning that a designer uses available tools (drawings, software) to convert ideas to a geometric representation. We never refer to those aspects of design which touch cognitive science or artificial intelligence.

\section{Overview of the paper}

This paper is a survey, discussing a wide range of topics. It is divided into sections as follows: §2: Flat panels; §3: Developable panels; §4: Smooth double-curved skins; §5: Paneling; §6: Support structures; §7: Repetition; §8: Patterns; §9: Statics; $\S 10$ : Shading and other functional aspects; $§ 11:$ Design exploration. Within each section we address the following points:

- We point out why a certain topic gets addressed and which practical aspects are motivating it.

- We discuss the most essential and interesting aspects of the methodology for its solution.

- Results are provided along with a discussion which is based on real projects wherever possible.

- We address open problems and directions of future research.

We tried to make this survey as self-contained as possible. However, some background on geometry processing, optimization and elementary differential geometry is necessary. For a very simple presentation of the geometry background along with a path towards ongoing research in Architectural Geometry, we refer to [77]. For collections of publications related to the field, we especially point to the volumes [21], [43] and [11].

\section{Polyhedral surfaces - structures from flat panels}

In order to realize a freeform surface in architecture, one often breaks it into smaller elements, called panels. Certainly, flat panels are the easiest and cheapest to produce and thus surfaces composed of flat panels, so-called polyhedral surfaces or polyhedral meshes play a key role in Architectural Geometry. In this section, we discuss their various types, with a focus on meshes from planar quads. They have turned out to be the most interesting species of panel from the viewpoint of mathematics.

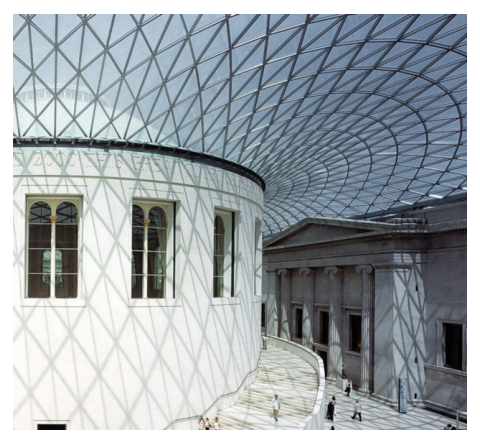

Figure 2: Triangle meshes in freeform architecture. Laying out a triangular pattern on a freeform surface and controlling its density and flow is a challenging problem successfully solved in 2000 by Chris Williams for the Great Court Roof of the British Museum in London (designed by Foster+Partners). Here dynamic relaxation was employed to aesthetically distribute the triangles (Photo: Waagner-Biro Stahlbau).

\subsection{History of polyhedral surfaces in architecture}

Until the 20th century, polyhedral surfaces in architecture appeared predominately as rotational surfaces used for roof and dome structures. Quads were the base polygon of choice for most of these early examples, mainly due to their aesthetic qualities and their economic advantages.

In 1928, engineers of the Carl Zeiss optical company used a triangulated spherical dome structure for a planetarium in Jena, Germany. The triangulation was derived by projecting the vertices of a platonic solid with regularly triangulated faces to a sphere. 20 years later, R. Buckminster Fuller reinvented, developed and popularized this concept under the name of "geodesic dome". The increased stability of triangular elements compared to quads was one of the advantages of geodesic domes.

Later, Computer-Aided Design, by augmenting and replacing the more traditional tools for modeling freeform geometry, not only enabled more complex structures, but even created a demand for them. Designers suddenly had significantly more powerful design tools at their disposal, which in turn drove engineers to meet the challenge and again provide means of costeffective production. Last, but not least, the gap between design freedom and production was the main driving force for mathematicians and computer scientists to found the interdisciplinary research field of Architectural Geometry.

We discuss the newer developments regarding meshes and free forms below, starting with triangle meshes.

\subsection{Triangle meshes from the architectural perspective}

Triangle meshes are ubiquitously used in Graphics. Their use in architecture has different reasons and different aspects become important. We list some of them:

- Edges are often visible and smoothness (or lack of smoothness) of mesh polylines is an important part of the design. This is in contrast with geometry processing and computer graphics, where triangle meshes serve as amorphous representations of shapes and the orientation of edges hardly plays a role. 


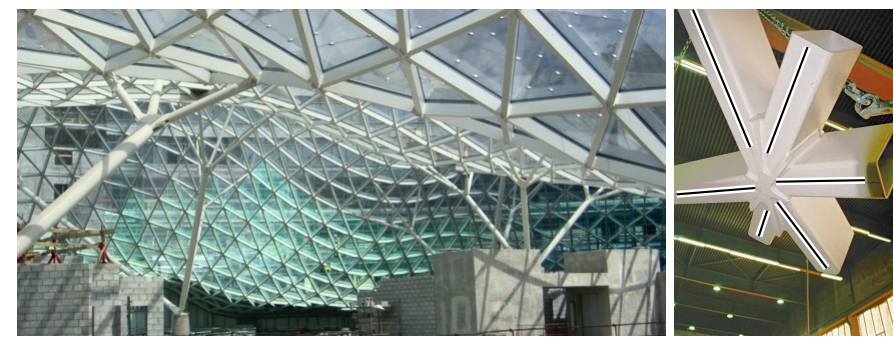

Figure 3: Complexity of nodes in triangle meshes. This image shows part of the roof of Zlote Tarasy in Warsazaw, designed by The Jerde Partnership, which is based on a triangle mesh, and a detail of a connector element between beams. The highlighted central lines on the beams demonstrate clearly that this is a vertex with torsion, i.e., the beams are in general position relative to each other (Photos: Waagner-Biro Stahlbau).

- The same is true for the placement of singularities (nonvalence 6 vertices). The combinatorics (i.e., connectivity) is to be chosen in line with the architect's vision. The designer will need tools for connectivity editing such as [62].

- There might be side conditions like the alignment of edges with other elements (e.g. floor slabs).

- There may be restrictions on the size of panels (for instance, the strict upper bound furnished by the biggest glass panels which are available, or the soft lower bound presented by the aim for lightness of the structure).

- Standard methods of designing meshes like subdivison may not provide enough flexiblity for mesh resolution control.

- The unit normal vectors of faces constitute the Gauss image of the mesh, which is inscribed in the unit sphere. The aesthetic quality of the Gauss image is observed indirectly, as oscillations in the surface; its algorithmic treatment is analogous to the treatment of the mesh itself.

Of course these aspects are true in general, not only for triangle meshes. We illustrate these questions with descriptions of real projects in $\$ 2.7$.

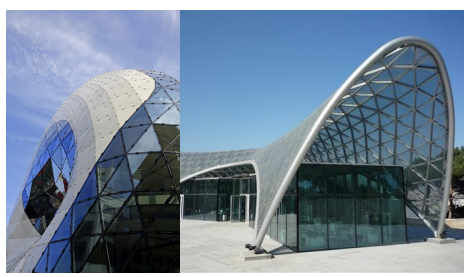

Figure 4: Optimization of triangle meshes: The Blob (left, photo by Evolute) in Eindhoven underwent Laplacian fairing subject to constraints like following floor slabs. Similar techniques were used for the Baku Funicular in 2012 (right, photo by T. Lorenz ZT).

The main point in favor of using triangle meshes to represent freeform shapes is the ability to freely move vertices for design purposes without having to worry about the side-condition that panels remain planar. Furthermore, triangle meshes have good structural properties. Triangular panels also have some disadvantages. Firstly, cutting typical triangular panels from rectangular sheets tends to generate more waste than cutting a typical quadrilateral panel. A more substantial disadvantage is that in a triangle mesh, a typical vertex is incident with 6 edges and thus is significantly more complex than the valence 4 vertices typical for quad meshes (see Fig. 3). Generally triangle meshes require more parts and are heavier than quad meshes.

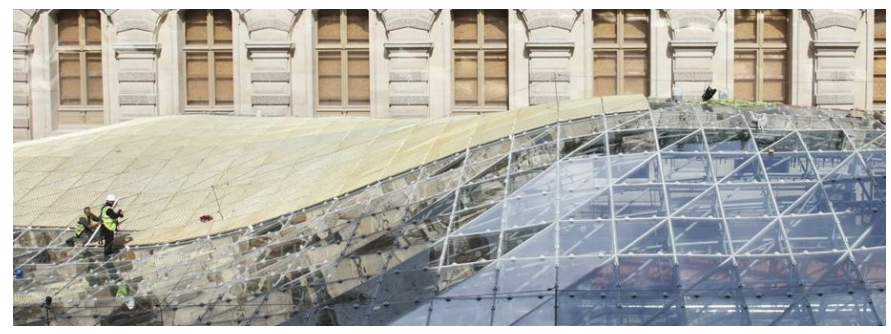

Figure 5: Pros and Cons of triangle meshes: The roof of the Islamic art exhibition in the Louvre, Paris, by Mario Bellini Architects and Rudy Ricciotti, is based on a hybrid triangle-quad mesh, combining the many degrees of freedom of triangle meshes with the lightness and smaller number of parts of a quad mesh. Photo: Waagner Biro Stahlbau.

A compromise between these pros and cons might be furnished by hybrid meshes, see Fig. 5 .

\subsection{Planar quad meshes}

Meshes from planar quadrilaterals ( $P Q$ meshes) do not have the disadvantages of triangle meshes mentioned above. Early projects by Schlaich, Schober and others [92, 37] use special types of PQ meshes whose faces are parallelograms or trapezoids and which are discrete versions of surfaces with a simple kinematic generation. We refer to Fig. 6 for the Berlin "hippo house", and we also mention the Sage Gateshead music hall. While representing striking designs, these shapes are not truly freeform, because they are generated by sweeping a curve and are therefore intrinsically one-dimensional. They are not the result of designing a two-dimensional surface in space.

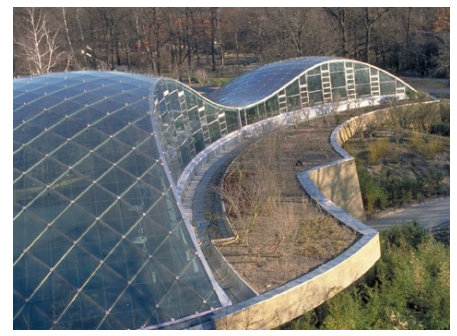

Figure 6: PQ meshes of simple geometry. Parallel translation of a polyline along another polyline generates a quad mesh with parallelogram faces (Hippo house, Berlin zoo, 1996. Photo and Engineering: Schlaich Bergermann \& Partners).

\subsubsection{Differential geometry of planar quad meshes}

General PQ meshes were first addressed in Discrete Differential Geometry $[84,16]$, where they appear as discrete counterparts of conjugate curve networks. Let us give the definition: Assume that $\mathbf{s}(u, v)$ is a parametrization of a surface. With the unit normal field $\mathbf{n}=\left(\mathbf{s}_{u} \times \mathbf{s}_{v}\right) /\left\|\mathbf{s}_{u} \times \mathbf{s}_{v}\right\|$, we define the coefficient matrix $\left(\begin{array}{cc}L & M \\ M & N\end{array}\right)=\left(\begin{array}{lll}\mathbf{s}_{u u} \cdot \mathbf{n} & \mathbf{s}_{u v} \cdot \mathbf{n} \\ \mathbf{s}_{u v} \cdot \mathbf{n} & \mathbf{s}_{v v} \cdot \mathbf{n}\end{array}\right)$. Vectors tangent to the surface in a point $\mathbf{s}(u, v)$ are described as linear combinations of the partial derivatives: $\mathbf{t}_{1}=u_{1} \mathbf{S}_{u}+v_{1} \mathbf{S}_{v}$ and $\mathbf{t}_{2}=u_{2} \mathbf{S}_{u}+v_{2} \mathbf{s}_{v}$. We say that these two vectors are conjugate, if the second fundamental form on them evaluates to zero: $\operatorname{II}\left(\mathbf{t}_{1}, \mathbf{t}_{2}\right)=L u_{1} v_{1}+M\left(u_{1} v_{2}+u_{2} v_{1}\right)+N u_{2} v_{2}=0$. It is also well known that the principal curvature directions are those conjugate directions which are at the same time orthogonal (see $\$ 2.4$ below).

To derive a network of conjugate curves on a given surface, one can start with an arbitrary family of curves covering the 
surface. In each point of the surface, pick a vector $\mathbf{t}_{1}$ tangent to that family of curves, and compute $\mathbf{t}_{2}$ by solving the linear system $\operatorname{II}\left(\mathbf{t}_{1}, \mathbf{t}_{2}\right)=0$. Finally integrate the vector field $\mathbf{t}_{2}$ to get another family of curves which is conjugate to the first one.

This procedure unfortunately in practice does not work nearly as well as the theory suggests, because many networks of conjugate curves are not useful as a guide for edges of a quad mesh. Problems occur in connection with self-conjugate (asymptotic) directions of which there are two in each hyperbolic surface point. This is the reason why PQ remeshing of a given surface is especially difficult in negatively curved regions. The danger to obtain quads with very small angles is high unless one is close to the principal directions. As a consequence, one is almost forced to use the principal curvature lines to guide a quad remeshing, and almost no design freedom ist left. This topic is explored in more depth by Liu et al. [65] and Zadravec et al. [116]. We also refer to A. Schiftner [85] who uses relative principal directions.

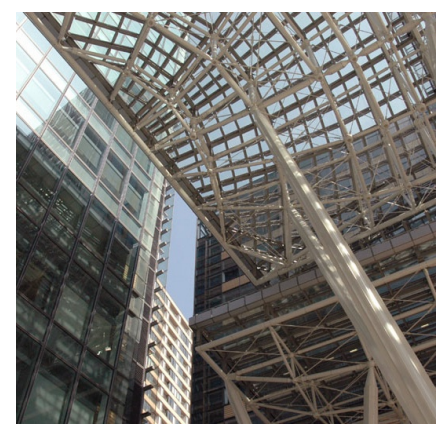

Figure 7: Example of a freeform PQ structure: The Roppongi Canopy at Tokyo Midtown Plaza, realized in 2005 by Buro Happold. Little documentation about this project is publicly available, but it appears to be based on a planar quad mesh following the principal curvature lines of the design surface.

\subsubsection{Computing Planar Quad Meshes}

The design and computation of PQ meshes have first been addressed in full generality by Liu et al. [64]. They based PQ mesh computation on nonlinear optimization, with face planarity as nonlinear constraint, and with an objective function which considers fairness of the main mesh polylines and - if applicable - proximity to a given reference surface or boundary curves. Optimization will fail, however, if it does not start from an initial mesh which is already close to planar and which, in view of the corresponding theory, is not close to a conjugate curve network covering the design surface. As an effective design method circumventing this problem, the authors propose to alternate between a quad-based subdivision scheme and optimization to maintain planarity of faces throughout all scales.

\subsection{Nearly rectangular panels, support structures and offsets}

It is natural to ask for PQ meshes whose faces are as close as possible to rectangles. This would probably generate the least waste when cutting the panels, as most materials come in rectangular shapes. Moreover, one does not have to deal with too small angles. As it turns out, such meshes are discrete versions of the principal curvature lines (which is a topic well known in Discrete Differential Geometry, see [16]). This connection is visible e.g. in the mesh of Fig. 7, whether intentional or not.
There are two major types of "principal" meshes: Circular meshes were first addressed by R. Martin et al. [67] (without regard to freeform architecture) and are defined by all faces having a circumcircle. Liu et al. [64] introduced conical meshes where all vertices are conical. This means that each vertex has a right circular cone which is tangent to the faces which meet there. The geometry of these meshes is remarkable:

- Alternative definitions involving angles between edges may be given: If $\alpha_{1}, \ldots, \alpha_{4}$ are the angles between edges in the vertices of a face, then essentially this face has a circumcircle $\Longleftrightarrow \alpha_{1}+\alpha_{3}=\alpha_{2}+\alpha_{4}=\pi$. If $\omega_{1}, \ldots, \omega_{4}$ are angles between successive edges emanating from a vertex, then this vertex is conical $\Longleftrightarrow \omega_{1}+\omega_{3}=\omega_{2}+\omega_{4}$.

- Conical meshes are characterized by existence of an offset mesh at constant face-face distance. Analogously, for quad meshes we have the equivalence of circularity and existence of an offset mesh at constant vertex-vertex distance. In both cases, the correspondence of original mesh and offset mesh is so that not only corresponding faces are parallel, but also corresponding edges (parallel meshes, cf. [80]).

For freeform architecture in particular the conical case is relevant, e.g. when realizing freeform shapes with plates of constant thickness and perfect alignment - see [79] and Fig. 8. There are close relations between offset properties of meshes and the torsion-free support structures of $\S 6$.
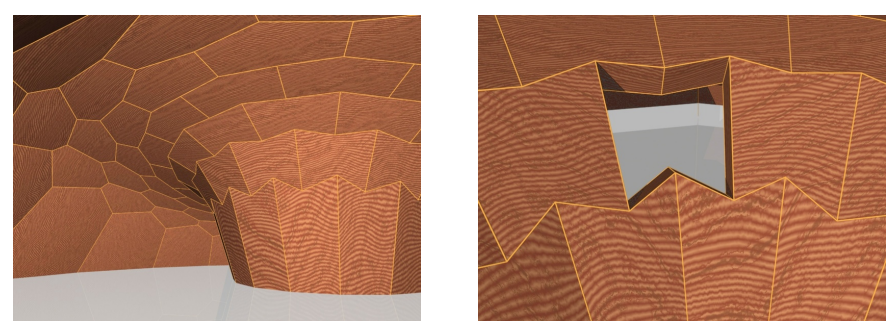

Figure 8: Offset properties of conical meshes. This hex mesh has planar faces and an offset at constant face-face distance, meaning that it represents the outer and inner boundaries of an arrangement of panels of constant thickness (image taken from [79]. Note: This particular mesh trivially enjoys the conical property, since all vertices have valence 3 .

Remark on panel shapes. It turns out that quad meshes whose faces are nearly squares (or rectangles with fixed edgelength ratio) are able to approximate only shapes which are contained in the catalogue of "isothermic" surfaces. This includes the minimal and soap bubble surfaces. We do not go into details, but only mention the work by Sechelmann et al. on quasi-isothermic mesh layout [93].

\subsection{Polyhedral hex-dominant meshes}

Hexagonal meshes (more precisely, meshes of vertex valence 3 ) are challenging to design. The low vertex degree mandates the use of varying and non-convex face shapes in order to capture different curvature regions. In addition, such meshes are more rigid and not as susceptible to deformation and planarization methods as quad meshes might be. The most direct approach 
to design such meshes is by means of the duality to triangle meshes. One approach to leveraging this duality is to use the unit normal vector field to map the surface to the unit sphere (i.e., the Gauss image), triangulate the image domain on the sphare, and map that triangulation back to obtain a planar-hex meshing of the original surface, cf. [1]. While this approach is simple, it is quite difficult to control the shapes of faces created in this way. Another approach is to assign a single plane for each primal vertex of a triangle mesh, and then the dual vertices are obtained by the intersection of these planes [102, 103]. Variational approaches can be applied in order to control the location of intersection vertices, by establishing proper plane coefficients [118]. However, the dual approach couples the topology and the quality of the resulting hex mesh with that of the primal triangle mesh.

Thus, Li et al. [121, 122] propose to remesh the triangle mesh into an "ideal triangulation", oriented with the principal directions. This triangulation produces dual planar-hex meshes with some guarantees on the quality of face shapes and approximation in non-parabolic regions. Specifically, [121] handles singularities by first producing a quad mesh aligned with principal curvatures, triangulating the mesh by diagonal splitting, and then taking the dual of this triangulation. In addition, they handle principal singularities of degree $\pm \frac{1}{2}$ by splitting into smaller 7-gons and 5-gons, and add corrections for parabolic lines which are not curvature lines in order to control hexagonal shapes between curvature regions. However, the algorithm requires some manual adjustments, such as the extraction of parabolic lines.

Other variational approaches, e.g. [23], seek to produce general polyhedral meshes that approximate the surface without necessarily being hexagonally-dominant or structured. Approximation is done by choosing a partition of the surface into patches. These patches are found by segmenting the shape according to a shape metric that measures normal variation and deviation from planarity. Thus, planar faces are considered a good approximation to these patches.

\subsection{Computational methodology}

Achieving geometric properties like planarity of faces as a rule has to be done by global optimization or a related procedure. Various numerical approaches have been used: constrained minimization, nonlinear least squares, penalty methods, augmented Lagrange methods, and others. For computing polyhedral surfaces, we mention [64], the work by R. Poranne et al. [76, 75], the projection method of Bouaziz et al. [20], the augmented Lagrangian method of Deng et al. [25], and the guided projection method of Tang et al. [100]. This list is not exhaustive.

Frequently it has been the case that such a numerical method, if used as a black box, would not succeed. Initialization proved very important. Usually, understanding a continuous analogue of the discrete objects under consideration is key for initialization, as described in $\$ 2.3 .2$ for the special case of quad meshes with planar faces. We return to computation in $\S 11$.

\subsection{Real projects}

The images in this section already show an overview of projects based on polyhedral surfaces which have been realized. As to triangle meshes, let us again point to Figure 2 (great court roof, British museum) for a mesh optimized for aesthetics, and to Figure 4 (the Blob) for a mesh optimized for floor alignment in addition to aesthetics. The undisputed advantages of quad meshes over triangle meshes mentioned above have led to projects based on PQ meshes: Fig. 6 shows an example of restricted design freedom, while Fig. 7 illustrates the first example of a freeform PQ mesh. Despite the fact that research in Architectural Geometry has provided various tools for layout and optimization of PQ meshes, still very few real projects use them (see however Fig. 33 for the Eiffel tower pavilions which are based on the same theory). For hybrid meshes which try to combine the advantages of both quadrilateral and triangular surfaces, see Figure 5.

\subsection{Open problems and research directions}

The following list is a collection of problems which are unsolved and of situations which are not fully understood at the moment.

- Rationalization with PQ meshes is still a challenge as we are lacking a good overview of all suitable PQ remeshings (only those PQ meshes which resemble a smooth curve network are well understood via their connecton to conjugate curve networks).

- PQ remeshing becomes more difficult if constraints on panel sizes and shapes are imposed. Very little is known on how to properly properly initialize optimization algorithms if such side conditions are present (initialization includes the choice of mesh combinatorics, which is relevant to the success of optimization as well as for aesthetics).

- In our research on polyhedral meshes, good results were obtained with objective functions which try to achieve nearly equal panel areas. However, we are not aware of results concerning the smooth limit of the quad mesh case. Such a smooth limit would be an area-preserving conjugate parameterization (i.e., a parametrization where the determinant of the first fundamental form is constant, and the second fundamental form is diagonal). Knowledge in this area would help with remeshing.

- There has been progress on quad patch layout algorithms, see e.g. [18, 29]. It would be interesting to use similar ideas for PQ remeshing by addressing the problem in a more global way (in contrast to local considerations using conjugate vector fields). Even a rather coarse PQ remeshing would mean significant progress, since subdivision combined with optimization can be employed for refinement. Automated initialization of subdivision-based approximation is currently not well understood, even without the additional constraint of planarity of faces.

- A number of open problems arise from the combination of shape design with aspects of statics (see §9). This applies to polyhedral meshes, but also to other kinds of shape representations. 
- Many additional criteria, e.g. related to energy performance or fabrication play a role in practice. This shows that in this area, like elsewhere, there is much demand for robust, fast, and efficiently usable multi-criteria optimization tools (for initial results see $\S 11$ )

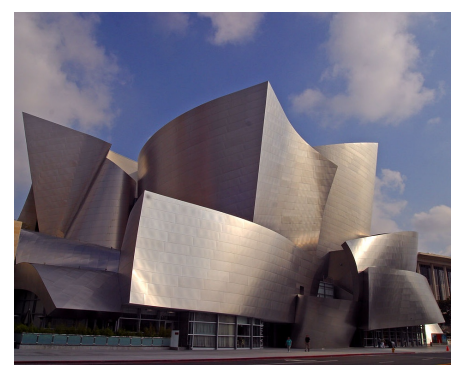

Figure 9: A pioneer of using developable freeform surfaces is Frank Gehry. A combination of physical and digital modeling has been used for the 1997 Guggenheim Museum in Bilbao as well as for other projects like the 2003 Walt Disney Concert Hall in Los Angeles (at left, photo by Jon Sullivan).

\section{Developable panels and semi-discrete models - skins from smooth strips}

\subsection{Developable surfaces as limits of $P Q$ meshes.}

Developable surfaces, also known as single-curved surfaces, can be unfolded into the plane without stretching or tearing. Thus it is easy to cover them with panels from metal or other materials with a similar behavior. They are characterized by containing a family of straight lines, each of which possesses a constant tangent plane - see Figure 10 for an illustration of this fact and a limit process which transforms a sequence of planar quads into a developable strip. Their developability is not the only property of such surfaces relevant to fabrication: also the straight lines contained in developables have advantages for the fabrication of the substructure.
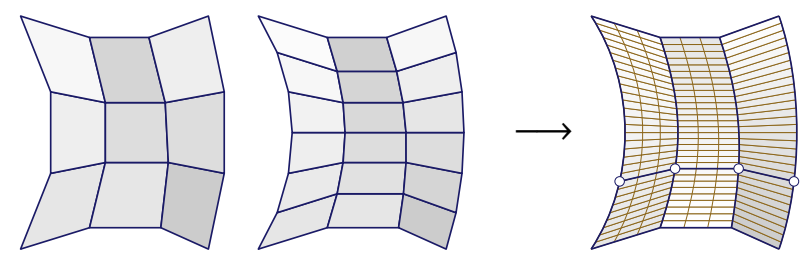

Figure 10: Generating developable strip models by refining a PQ mesh so that the quads stay planar in all stages. In the limit (right) one gets ruled strips with a constant tangent plane along each ruling (namely, the limit of a face plane in the PQ strip).

The work of Frank Gehry (Figures 1, 9, 11) is an excellent example for the use of developable surfaces in architecture and for the influence of digital technology. The metal cladding in early projects is less perfect than the one in later projects where Gehry could already use specialized software for representing developable surfaces, integrated by Gehry Technologies into the CAD system CATIA [94].

The limiting process that generates a developable strip from a strip of planar quads, may also be applied to an entire PQ mesh (see Figure 10). The result is a D-strip model [81]. This piecewise developable surface can be seen as a semidiscrete surface, smooth in one parameter direction and discrete in the other one. Computationally, one may not want to work with a representation which exhibits very thin quads. It is better to represent the individual strips as B-spline surfaces of degree $(1, n)$ (usually $n=3$ ) and optimize them towards developability [81]. For the rather large literature on developable spline surfaces, see e.g. $[3,22,83]$.

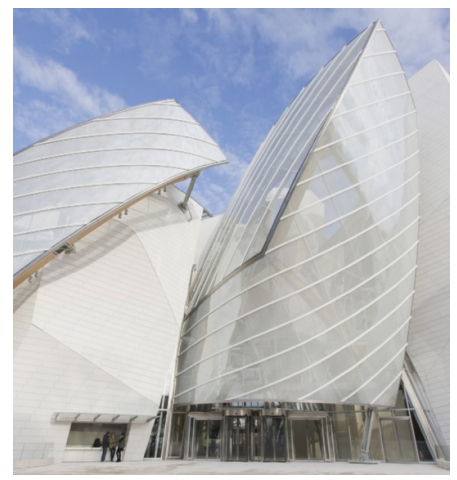

Figure 11: Each of the 'sails' of the Fondation Louis Vuitton by Frank Gehry consists of developable strips - for a mathematician this is an example of a semidiscrete conjugate surface. For the actual building, the strips were approximated by cylindrical glass panels (Photo: Mairie de Paris).

\subsection{Special cases of strip models}

The strip boundaries and the rulings across the strips represent a network of curves (much like the edge polylines in a regular PQ mesh) which is known to be a semidiscrete version of a network of conjugate curves. Special instances of conjugate networks correspond to special kinds of D-strip models. For example, imposing the condition of orthogonality yields the principal curvature lines, whose semidiscrete counterparts have properties similar to the circular and conical meshes encountered earlier [81]. One of them is the existence of a semidiscrete support structure which is important for fabrication and which is visualized in Fig. 33: There is a developable approximately orthogonal to the surface through each strip boundary; such developables may serve as the sides of curved beams with rectangular cross-section which are manufacturable by bending, cf. $[89,4]$. We treat this topic in detail in $§ 6.1$.

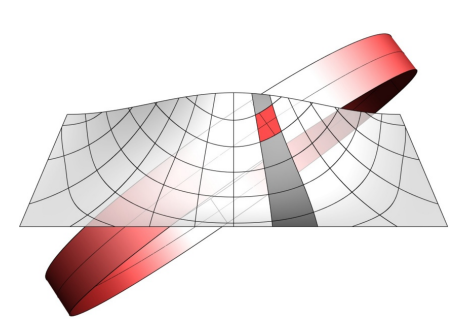

Figure 12: The Eiffel Tower Pavillons feature near-deveopable strips; it follows that this design essentially represents a semidiscrete conjugate surface. That property also led to a very good rationalization of the developable glass strips by cylinders. The image shows the design with one cylinder belonging to a glass panel.
Another special case are geodesic D-strip models, whose strips follow geodesic curves, modeling straight strips of paper which are put onto curved surfaces. They have interesting applications in paneling with wood. Since wooden strips are easily subject to torsion, they can be modelled as developable surfaces to a lesser degree than skins made from sheet metal. Wooden panels in the Burj Khalifa (2010, Dubai) provide an example, see Figure 13 and [69]. 

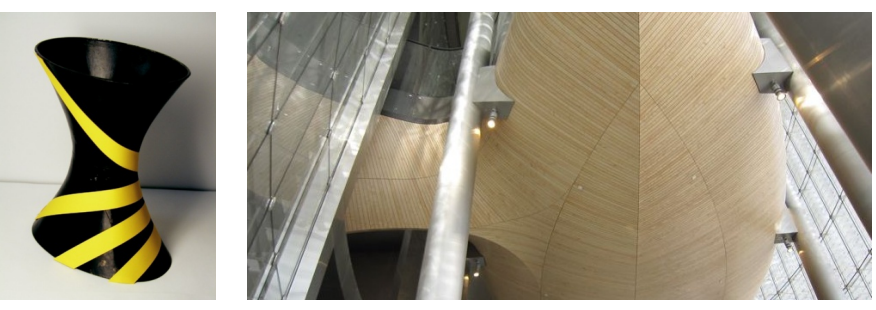

Figure 13: Geodesic strips. Putting straight bendable strips onto a surface yields a pattern which follows geodesic lines (left). The image at right shows a paneling of a freeform ceiling (Burj Khalifa, design by Gehry Technologies) which is guided by this principle.

\subsection{Curved folding}

The planar developments of the individual strips of a D-strip model do in general not fit together. However, there are developable surface strips which are glued together in a way such that the development keeps them together. Here one speaks of a curved fold. Designs made via curved folding may have a striking elegance and have been computationally addressed via PQ strips within an appropriate optimization framework by Kilian et al., see [58] and Fig. 14.
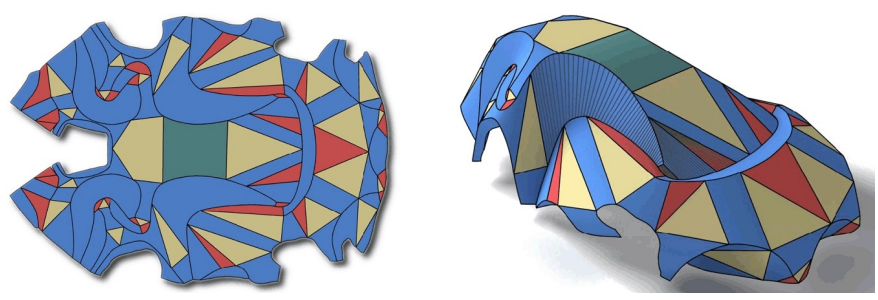

Figure 14: Curved Folding. Folding paper along the pattern outlined at left creates the "car" surface at right. Colors indicate the various types of developables which occur: red for cones, green for cylinders, light brown for planar patches, and blue for general developables (image courtesy M. Kilian).

\subsection{Technology for single-curved skins and real projects}

Various technologies are used to produce single-curved skins. An important part is played by the ability of CAD systems to handle such surfaces. Regarding materials, sheet metal is popular (cf. Disney concert Hall by Gehry, Fig. 9). For recent developments in industrial processes see e.g. the paper [91] by Bemo systems.

Glass as a material is more challenging than metal because of the high cost of hot bending glass into curved shapes. An interesting technology is cold bending of laminated glass. Cold warping, in the literal sense, of laminated units up to or exceeding the tolerances guaranteed by manufacturers has been employed for quite some time; the IAC building in New York by Frank Gehry is an example of this. A later innovation is to apply the laminating process, involving $130^{\circ} \mathrm{C}$ heat and $10 \mathrm{bar}$ pressure, to pre-bent glass. This was done first on a large scale by RFR for the 2007 Strasbourg train station (Fig. 16).

Another approach rests on the availability of cylindrical glass panels: Single-curved skins, approximately decomposed into cylindrical panels, have been employed for the Fondation Louis Vuitton (Fig. 11) and the Eiffel tower pavilions (Fig. 33).
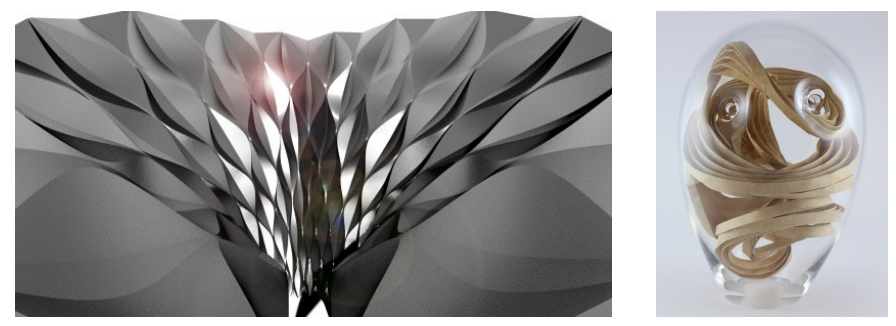

Figure 15: Curved Folding. Left: ARUM installation at the 2012 Venice biennale (Photo: Zaha Hadid press release). Right: A "waves in glass" sculpture by Erik and Martin Demaine.

Also wood is suited to realise developable shapes, which has long been utilized in ship building and the early aerospace industry. There seem to be no really large scale freeform structures using single-curved wooden panels. However we point to the "geodesic" paneling of the Burj Khalifa ceiling (cf. [69] and Fig. 13), and to the research done by the Laboratory for timber constructions at EPFL (see http://ibois.epf.ch).

The application of developable surfaces with curved folds is rare in architecture. An exception is the company Robofold which specialises on bending thin metal sheets with robots. A recent example of their work is ARUm installation for Zaha Hadid Architects (Fig. 15). That figure also shows other artwork involving curved folds.

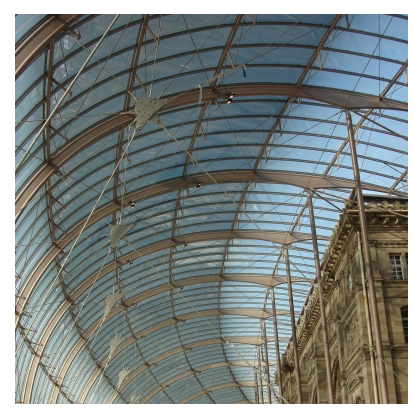

Figure 16: The so-called cold bending technology allows the production of general developable shapes from a single glass panel. The first large scale application of this technique was the 2007 canopy over the Strasbourg train station (at left, photo and engineering by RFR). Here panels are cylindrical, but the technique is applicable also to more general shapes (with the limitation of relatively large minimal bending radius compared to hot bending).

\subsection{Open problems and research directions}

Due to the theoretical proximity of PQ meshes and D-strip models, most open problems for PQ meshes also translate to the present setting. We add a few which are not direct counterparts and which are not yet mentioned in $\$ 2.8$.

- A general D-strip model is not yet useful for materials like glass. It is to be expected that for some time, producing cylindrical gass will remain much cheaper than producing general developable shapes, so the question of rationalization arises (see Figures 11,33). D-strip models whose individual strips can be well approximated by sequences of simpler panels such as cylinders, are an open question, especially rationalization with such structures.

- Digital design of objects via curved folding is pretty wide open. So far, mainly the digitial reconstruction of physical models and simple deformations have been addressed, and indeed curved folding itself has yet to be applied on a larger scale. 
- The interactive isometric deformation of developable surfaces is only easy if we restrict ourselves to those deformations which preserve rulings. To our best knowledge, there is no elegant handling of more general isometric deformations, apart from efficient simulations based on triangle meshes, cf. [97].

\section{Smooth double-curved skins}

Realizing a double-curved freeform surface as a large and completely smooth (tangent plane continuous) architectural skin is a great challenge, and for some materials is only affordable when deviating from perfect smoothness or when accepting restrictions on the possible shapes. In this section we only address perfect smoothness; the successful play with tolerances is discussed in connection with the paneling problem in $\S 5$.
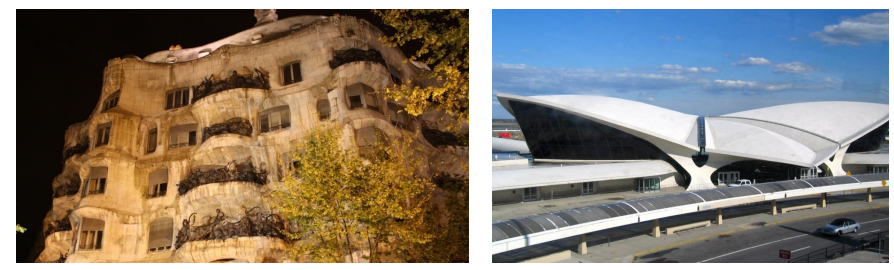

Figure 17: An early 20th century freeform geometry shaped in masonry is the 1910 Casa Milà in Barcelona by Antonio Gaudí (Photo at left by Kyle Taylor). Later, freeform shapes were realized at a grander scale in concrete, such as the TWA flight centre at JFK airport by Sarinen in 1962 (right).

Obviously, we need smooth double-curved panels which for most materials require the fabrication of a mould. There are only very few instances of double curved surfaces which can efficiently be produced. The most promising among those are ruled surfaces. Their production is rather easy in fibre-reinforced concrete, because the required moulds can be fabricated with hot wire cutting from styrofoam. However, they are only suitable to model negatively curved surfaces (\$4.3). On arbitrary surfaces, we so far know no better approach than using surface patches composed of (possibly congruent) circular arcs (\$4.4).

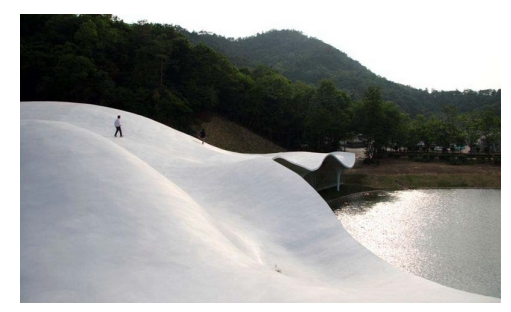

Figure 18: The 2006 Kakamigahara funeral hall by Toyo Ito is a freeform concrete shell without panels, still representing an instance of the "paneling problem" with respect to the formworks needed for pouring concrete (photo: Toyo Ito \& Associates).

\subsection{History and technology of double-curved freeform shapes}

Building beyond traditional forms has long been the aim of architects, and engineers. In the late 19th century, industrialization led to a more systematic approach to the problem, and new tools became available. In the second half of the 20th century, greater scale and complexity were achieved with concrete shells. See Fig. 17 for some such earlier examples. Shapes which are apparently "freeform" were frequently achieved by physical principles, e.g. Heinz Isler's thin shells constructed on top of pneumatic pillows. Concrete remains a popular material, its limitations being weight and cost. Prominent recent examples are the Rolex Learning Center at EPFL (Lausanne), or the 2006 Kakamigahara funeral hall by Toyo Ito, see Fig. 18.

Another method (not dicussed here) to realise smooth double curved surfaces is via tensile membranes, again achieving form by means of physical principles.
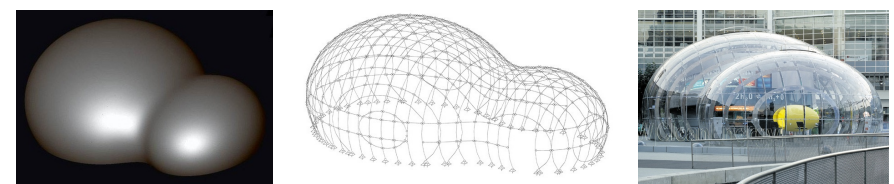

Figure 19: Digital work flow for the BMW 'bubble' design by Franken Architects (engineering and images: Bollinger and Grohmann).

With NURBS modeling becoming available in architectural design, the demand for cost-efficient realisations of smooth curved skins reached a new peak. One of the first true freeform shapes in a modern sense and one of the first projects that was completely created with digital means (from design to construction) is the 1999 BMW Bubble, see Fig. 19. Like the Kunsthaus in Graz (2003), it is cladded with acrylic glass. Also doublecurved glass panels have been used, see Fig. 20 for a shape which is truly freeform and Fig. 22 which is less so. The production of such panels by hot bending via molds can be quite expensive, especially if they are to form a watertight skin.

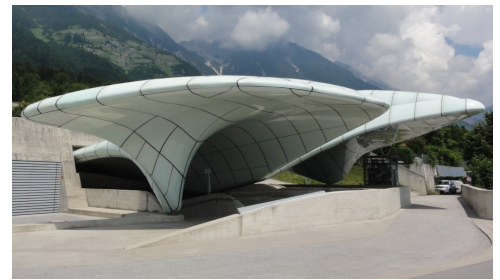

Figure 20: Double-curved glass panels with neoprene seals are covering Hungerburg station, one of a series by Zaha Hadid, built for the Innsbruck Hungerburgbahn in 2004 (photo: Wikipedia user Mattes).

More recently, CNC milled wood and double curved metal have been used to produce smooth freeform surfaces, such as the Kamppi Chapel, Helsinki 2012, or the Dongdaemun Design Plaza and Park. The Chicago Cloud Gate, see Fig. 21, is an artistic version of a "perfect smooth bubble".

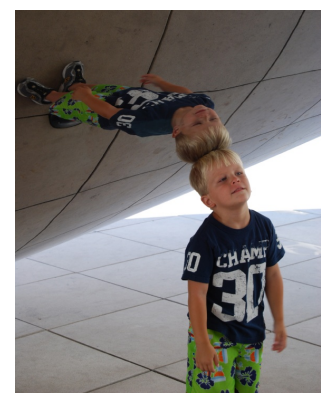

Figure 21: Reflections prove smoothness visually. The Cloud Gate sculpture by Anish Kapoor (Chicago 2006) has a seamless metal cladding (photo: Tim Schapker). Compare this image with Figure 23, where kinks in the reflection lines (at strip boundaries) reveal curvature discontinuities of the surface.

\subsection{Hidden repetitivity}

The high cost of producing double-curved skins has led to ideas how panels or molds used for manufacturing panels may be 
used more than once. A famous instance of this is the Sydney Opera House where concrete elements were produced with molds, and the overall spherical geometry ensured that molds could be reused. It should be noted that this was one of the first recorded instances of "computer programs" being used to solve problems of geometry, logistics, and structural analysis. On a smaller scale, but for more complex geometry and with different materials, this thought guides the shape of the 2003 lentille at Saint Lazare Metro Station in Paris, see Fig. 22.

For the cost-effective manufacturing of double-curved skins, hidden repetitive geometry is obviously highly relevant. Unfortunately it is very difficult to detect in general. We return to the topic of repetition - hidden or not - in $§ 7$.

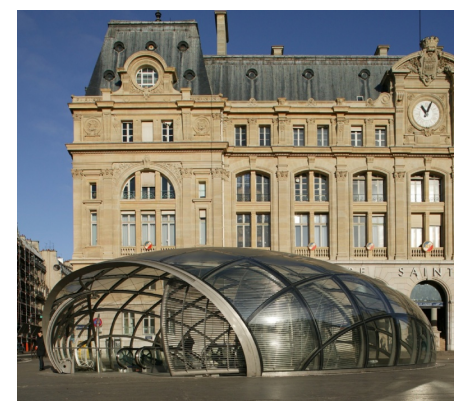

Figure 22: Repetition aides manufacturing efficiency. The lentille at Saint Lazare metro station is composed of a sphere and a torus, and has a rotational symmetry no longer apparent after panelization. This symmetry enabled the reuse of molds used in the manufacturing of glass panels (engineering and photo: RFR).

\subsection{Smooth negatively curved surfaces from ruled patches}

We here return to mathematical and algorithmic questions, discussing general smooth surfaces of negative Gaussian curvature and how they can be approximated by a smooth union of ruled surface strips. Such a representation is a semidiscrete model of a surface similar to the developable case discussed earlier in $\S 3.1$, but now with a different side condition: Individual ruled strips should join smoothly. The relevance of a ruled rationalization of a smooth surface for architecture lies in the fact that they are easy to manufacture.
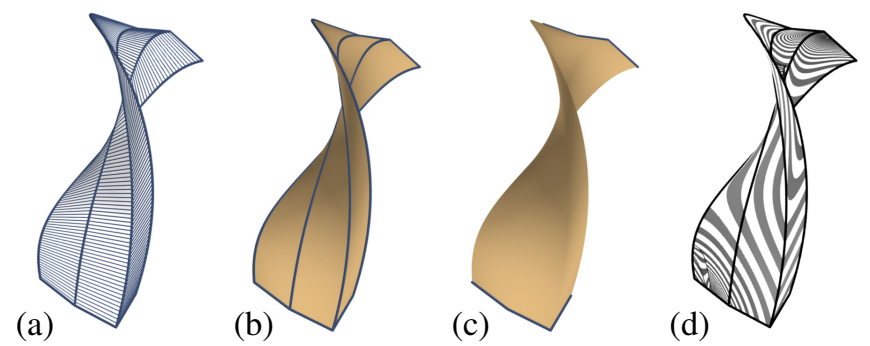

Figure 23: Ruled strips can join smoothly even if their rulings do not. This sequence of images shows 3 ruled strips with rulings (a), without rulings (b), both rulings and strip boundaries removed (c), and with visual proof of $G^{1}$ smoothness by continuity of reflection lines (d).

Figure 23 illustrates this situation: If successive strips have the same tangent plane in each point of the common boundary curve $c_{i}$, then successive rulings $p_{i-1} p_{i}$ and $p_{i} p_{i+1}$ must be coplanar with the tangent of $c_{i}$ in the point $p_{i}$. If the sequence of strips with this property is refined and converges to a surface of $C^{2}$ smoothness, then the polyline $\left\{p_{i}\right\}$ of rulings converges to a curve, and the plane spanned by 3 successive points $p_{i-1}, p_{i}, p_{i+1}$ will converge to the osculating plane of that curve. The polyline of rulings is therefore seen as a semidiscrete version of an asymptotic curve on the limit surface. This fact is used by S. Flöry et al. [32, 34, 33] for the initialization of algorithms for optimization, rationalization and design with smooth surfaces from ruled strips (cf. Fig. 24). If also strip boundaries correspond to asymptotic curves, then we obtain semidiscrete asymptotic parameterizations which have been investigated from a mathematical viewpoint by [110].
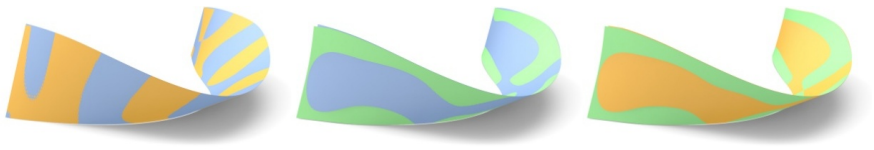

Figure 24: Approximation by ruled surfaces of different kinds: A reference surface (blue) which is an unmodified part of the Cagliari museum project by Zaha Hadid architects is approximated by Flöry et al. [33] with a conoidal ruled surface (green) and with a general ruled surface (orange). We show these surfaces superimposed onto each other in order to visualize approximation quality.

One can ask for smooth surfaces composed of especially simple ruled surface patches. Bilinear patches result in (smooth, but still discrete) models of so-called affine minimal surfaces and thus can only model special shapes [53]. However, smoothly joined rational bilinar patches (called hyperbolic nets) are sufficient to approximate arbitray simply connected surfaces. This has first been proved by Huhnen-Venedey and Rörig [48] using the Plücker quadric model of line geometry. A CAGD approach based on the rational Bezier representation and algorithms for remeshing are due to Shi et al. [95] (see Fig. 25). The patch boundaries of a hyperbolic net form an asymptotic net (A-net), i.e., a quad mesh with planar vertex stars. A-nets are well studied discrete asymptotic parameterizations [16].
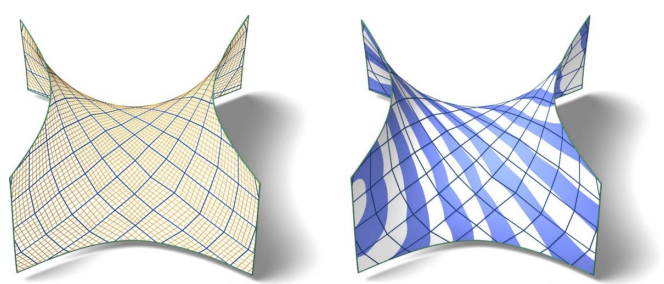

Figure 25: Negatively curved surfaces may be approximated by a smooth union of rational Bézier quads of degree 1-1. Left: Patches with rulings. Right: Continuity of isophotic curves proves $C^{1}$ smoothness, but non-smoothness of these curves reveals $C^{2}$ discontinuities.

Remark. Hyperbolic nets are remarkable structures in discrete differential geometry. Huhnen-Venedey and Schief [49] use them for the study of discrete Weingarten transformations, and there is ongoing research by W. Schief who employs them in a discrete theory of projective minimal surfaces.

\subsection{Smooth surfaces covered by circular arcs}

After ruled surfaces, the next step in complexity are surfaces covered by circles. Sophus Lie's sometimes mysterious linesphere correspondence [70] provides a direct way to convert surfaces carrying lines to surfaces carrying circles:

- Ruled quadrics (covered by 2 families of rulings) correspond to Dupin cyclides (envelopes of 2 families of spheres). 
- Asymptotic parameterizations correspond to principal ones

- Smooth surfaces composed of ruled quadric patches correspond to cyclidic nets covered by Dupin cyclide patches.

Cyclidic nets have been first addressed by R. Martin [67] and stimulated a lot of research on the use of Dupin cyclides in computer-aided geometric design. Only recently, Bobenko and Huhnen-Venedey [17] presented a complete theory and showed that every circular mesh can be extended smoothly to a cyclidic net. A computational treatment of these cyclidic nets along with generalizations aiming at substructures in freeform architecture has been studied by Bo et al. [14]. Even if cyclidic nets are covered by 2 families of simple curves (namely, circles), structures covered by circles of constant radius are more relevant to architecture. Patches which carry one family of congruent circles have been considered by Bo et al. [14]. Barton et al. [6] study the evolution of an arc spline curve (smooth curve composed of circular arcs) so that its arcs stay rigid but can rotate about the common tangents. This generalized sweeping concept is useful to design smooth freeform surfaces composed of congruent circular arcs, but exhibits restrictions in approximating arbitrary surfaces - see Fig. 26.
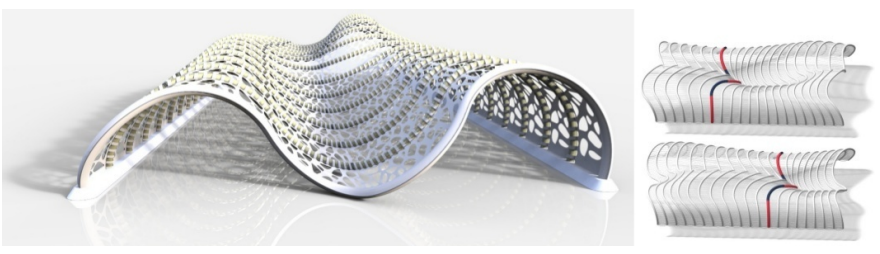

Figure 26: A non-static roof composed of many instances of the same flexible 6-snake of circular arcs. This object is composed of many copies of a single circular arc, which is relevant to the cost of manufacturing. The small figures show two different stages of flexion.

\subsection{Open problems and research directions}

For "real projects" we refer to Sections 4.1 and 4.2, and also to the discussion of paneling in $\S 5$. The theoretical work surveyed in this section has yet to be applied in practice. Open questions in this area are:

- The main question here is whether there are useful panels that can generate smooth architectural freeform surfaces beyond surfaces of negative curvature. It is probably related to new manufacturing technologies for panels.

- Promising research directions connected with this section are in Discrete Differential Geometry and based on discrete models which are actually smooth surfaces from simple patches. The example of hyperbolic nets already turns out to be useful in discrete projective differential geometry, and thus one would also expect cyclidic structures having an important role in discrete differential sphere geometry.

\section{Paneling and the quality-cost tradeoff}

\subsection{Geometric and algorithmic aspects of panelings}

The paneling problem refers to realizing double curved architectural freeform surfaces by rationalization, i.e., replacing the surface by a union of panels. This section treats paneling in the narrow sense, where the panels are curved, and are to approximate the reference shape smoothly, up to tolerances. Paneling can be seen as two tasks, which are not independent:

1. Segmentation of the reference shape into smaller pieces, which are called segments.

2. Approximation of each segment by a panel which represents a manufacturable shape, such that tolerances are obeyed.

One objective of segmentation is to enable a good solution of the approximation task, and on the other hand, approximation provides some information in how to better segment the surface. Maximising approximation quality by iteratively coupling segmentation and approximation was proposed in various variants under the name "Variational Shape Approximation", see e.g. [23, 113, 114].

In the architectural application, however, the geometric approximation quality is not the only, and not necessarily the most important objective. Since often the seams between panels are highly visible, the segmentation problem is related to the aesthetical pattern layout problem (see e.g. polygonal mesh layout, remeshing, and patch decomposition [73] or the work by Bommes et al. [18]). Visibility of seams implies that in practice, segmentation often is an architect's design decision and only the approximation part is treated algorithmically.

Eigensatz et al. [30, 31] propose a solution to this problem, consisting of the following loop:

(i) Assignment of a panel type (planar, cylindrical, custom) to each segment;

(ii) Approximation of each reference segment by a panel such that tolerances (kink angles, gaps) do not exceed previously specified thresholds;

(iii) Modification of the assignment and solving (ii) again, with the intention that cost is minimized. Here we have to take into account that manufacturing curved panels may require molds, and that re-using molds can significantly reduce costs.

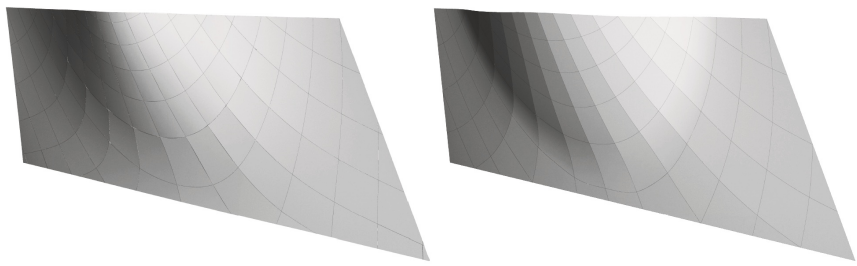

Figure 27: Panel optimization w.r.t. different targets: At left, the reference geometry of the Eiffel tower pavilions, cf. Fig. 33, is approximated by cylindrical panels. At right, the same approximation was performed with a bigger kink angle tolerance across strips, and a smaller angle tolerance to the upper and lower neighbours of panels (images: Evolute).

Subproblem (ii) is of a standard kind in numerical optimization, but is still challenging because if all degrees of freedom are exploited, it is global, nonlinear, and has many variables. Fig. 27 illustrated how this task was solved for the cylindrical panels of the Eiffel tower pavilions [89, 4]. 


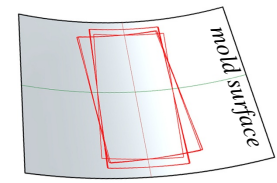

Figure 28: Cost-effective paneling: If curved panels (red) have to be made, they should at least be manufacturable from the same mold in order to save costs. This principle can apply to custom doublecurved panels as well as to cylindrical panels.

Subproblem (iii) contains a version of set cover; it is therefore provably hard, and finding the optimum is out of reach for today's computing power. The method of [30] has been applied to the paneling of the Arena Corinthians in Sao Paolo, cf. [87].
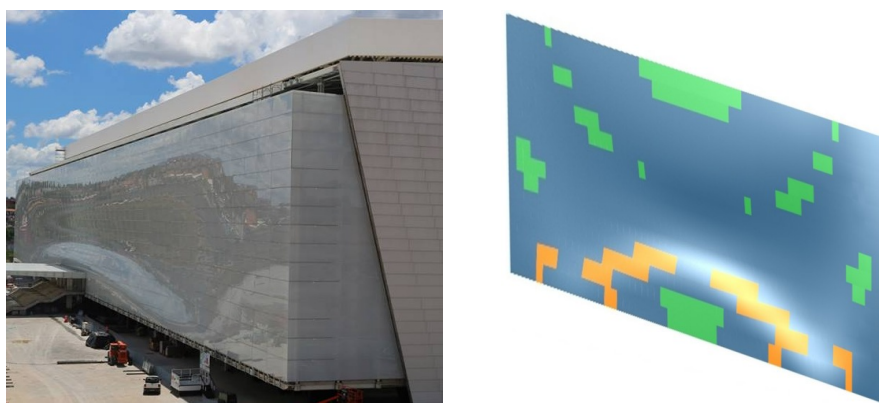

Figure 29: Combined combinatorial and numerical optimization of panelings. The facade of the Arena Corinthians in Sao Paolo (2014, architect: Aníbal Coutinho) is approximated by as few panel types as possible to keep manufacturing cost small. The prescribed tolerances $(6 \mathrm{~mm}$ of panel divergence and $5 \mathrm{~cm}$ deviation from the reference surface) were achieved by 855 panels made with 61 different molds. The right hand figure shows the types of panels used (green: planar panels; blue: cylindrical panels: other: double-curved panels). E.g. a set of 296 cylindrical panels could be made from the same mold, cf. [87].

The segmentation task also has structural implications. For example, the substructure is often aligned with the panel seams, directly assigning a structural role to the segmentation geometry. Besides some work on quad mesh layout (\$9), we are not aware of any work in architecture that scientifically addresses this link between segmentation and structural properties.

\subsection{Real projects}

Research on the architectural paneling problem is still rather young, and there are only few instances of freeform surfaces rationalised with curved panels. Cylindrical panels have been used for the Louis Vuitton Foundation (Figures 1 and 11) and the Eiffel Tower Pavilions (Figures 33 and 27) Similarly, the Manta Glasshouse at the Laverstoke Mill in Hampshire by T. Heatherwick (under construction) rationalises a double curved freeform surface with cylindrical glass panels that even serve a structural purpose.

Cost-optimized paneling, including mold reuse, was employed for the facade of the Arena Corinthians in Sao Paolo (see Fig. 29 and [87]), the metal paneling of the 2012 Atoll shopping centre by Formtexx, and the Middle East Centre at St. Antony's college in Oxford (under construction, by Zaha Hadid architects)

\subsection{Open problems and research directions}

- Given a freeform design, simultaneously optimize the layout of panel seams (maybe considering aspects of an aligned substructure) and the panels to achieve control over the solution which provides the best tradeoff in terms of meeting the design intent and the desired surface quality and staying within budget.

- Even the simpler problem of decomposing a surface into panels so that they fulfill important architectural constraints is a hard task. Research in this direction is probably also related to shape understanding on a larger scale and to shape segmentation. Using the knowledge which is indirectly present in a database of realized designs or directly generated models could be a possible approach (inspired by the segmentation method of Kalogerakis et al. [55]).

- For most non-flat panels (e.g., right circular cylinders) it is not known how to best arrange them in order to achieve as smooth as possible and aesthetically pleasing skins.

\section{Geometric support structures}

The term support structure can denote different things. In Sections 6.1 and 6.2 it denotes a technical term in geometry which is more properly called torsion-free support structure. We return to the general meaning of the word in $§ 6.3$.
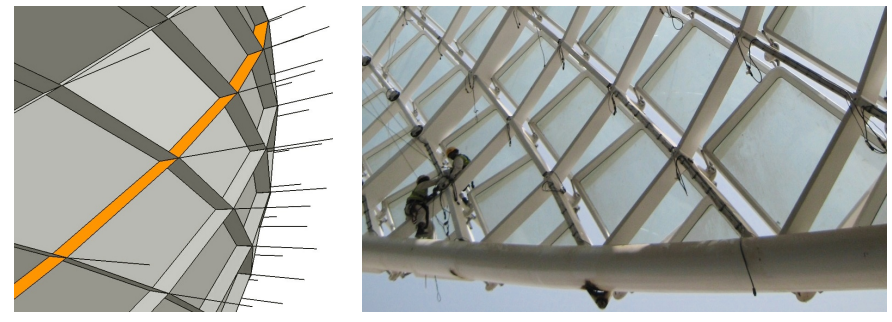

Figure 30: A geometric support structure (left, image by Evolute) is the basis of the outer skin built around the Yas island hotel, Abu Dhabi, designed by Asymptote Architecture (construction and photo at right by Waagner-Biro Stahlbau). The abstract support structure consists of an arrangement of quadrilaterals along the edges of a mesh. The actual beams follow those quads and intersect at nodes in a torsion-free way. The left hand image in particular shows the node axes and, highlighted in color, a developable strip of planar quads contained in the support structure.

\subsection{Torsion-free support structures}

Figure 30 illustrates this concept: We consider an arrangement of quads along the edges of a mesh, such that all the quads incident with a vertex do intersect in a common node axis. If we let the beams of an actual steel structure follow these quads, then they will not intersect in the unorganized manner of Figure 3. Rather they create an orderly "torsion-free" intersection as shown by Figure 31, where the symmetry planes of beams all pass through the common node axis. The list below shows a few instances of torsion-free support structures.

- Torsion-free support structures derived from offset meshes. We have already mentioned that two meshes might be at constant distance from each other. The vertex-offsets and faceoffsets mentioned in $\$ 2.4$ are special cases of a pair $M, M^{\prime}$ of meshes which have the same combinatorics, and which are positioned such that corresponding edges $e, e^{\prime}$ of $M, M^{\prime}$ are parallel to each other. In this case, each pair $e, e^{\prime}$ of edges spans a 


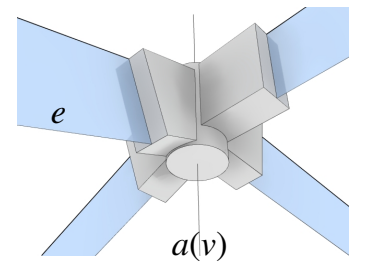

Figure 31: Detail of support structure associated with a mesh $M$. The support structure consists of the node axes " $a(v)$ " through every vertex $v$ and blue quads through every edge $e$. Aligning beams with the support structures yields torsionfree nodes.

quad of a torsion-free support structure, and each pair $v, v^{\prime}$ of vertices spans a node axis. For more details of this construction we refer to [80]. Figure 32 shows an example where $M, M^{\prime}$ are not only parallel, but even at constant face-face distance. This is possible because $M$ is a conical mesh (cf. §2.4); the node axes of the support structure in this case coincide with the axes of the cones associated with the conical mesh.

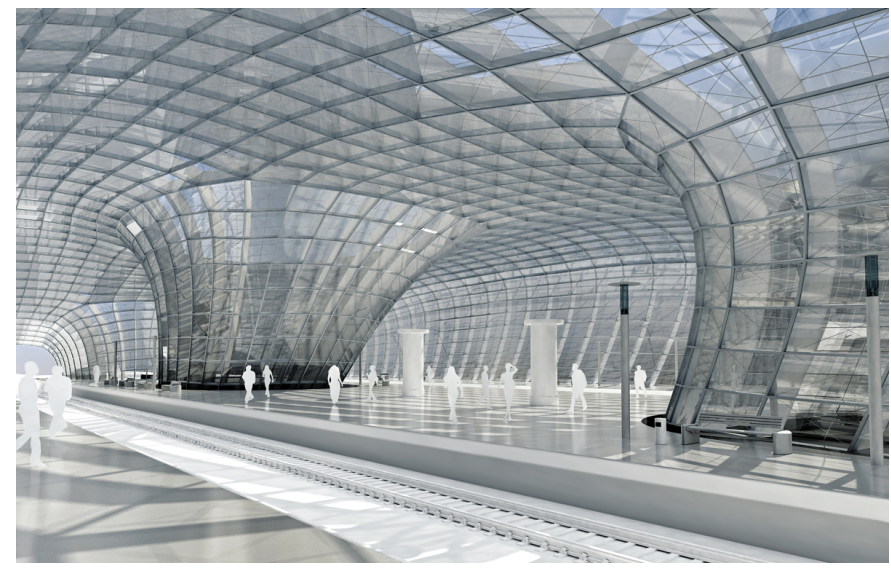

Figure 32: A pair of quad meshes with planar faces which are at constant faceface distance of each other. Both meshes $M, M^{\prime}$ are conical. In this rendering, the support structure which connects $M, M^{\prime}$ is materialized as quadrilateral elements which support planar glass panels. This image, created by B. Schneider, appeared on the front cover of the 2006 SIGGRAPH conference proceedings [64], and marks the establishment of "architectural geometry" as a separate discipline of geometry processing.

- Torsion-free Support structures derived from parallel polyhedral meshes. For any pair $M, M^{\prime}$ of offset meshes at distance $d$ we may define a Gauss image mesh $S=\frac{1}{d}\left(M^{\prime}-M\right)$ by vertexwise linear combination, which is again parallel to $M, M^{\prime}$. If the distance is measured between faces (resp., vertices) then $S$ is tangentially circumscribed to (resp. inscribed in) the unit sphere. Conversely, if $M, S$ are given, $M^{\prime}$ is reconstructed as $M^{\prime}=M+d S$. This construction establishes a link to discrete differential geometry. $S$ is a polyhedral surface playing much the same role as the continuous Gauss image, i.e., the unit normal vector field, in continuous differential geometry [80, 15]. It applies not only to the two special kinds of offsets mentioned here, but to all cases where meshes $M, M^{\prime}$ are parallel and at approximately constant distance from each other. The vertices of $S$ represent a collection of normal vectors associated with the corresponding vertices of $M$.

- Semidiscrete torsion-free support structures. Quad-mesh based support structures contain two families of developable strips, each constituting of a sequence of quadrilaterals (one of them is highlighted in color in Figure 30, left). One can imagine a limit process where the support structure becomes finer and finer, with ever smaller and denser quads, similar to Fig. 10. In the limit, the developable strips will become smooth developables. Selected developables from such a semidiscrete support structure can be found in the curved structural members of the Eiffel tower pavilions, see Fig. 33. The geometric considerations which lie at the bottom of this construction are detailed in $[89,4]$. In short, since each flank of each beam is to be made by bending, it has to be developable. Since the side flanks should be orthogonal to the reference surface, known facts from differential geometry imply that the beam should follow a principal curvature line. In summary there is hardly design freedom left, after the reference surface is chosen.

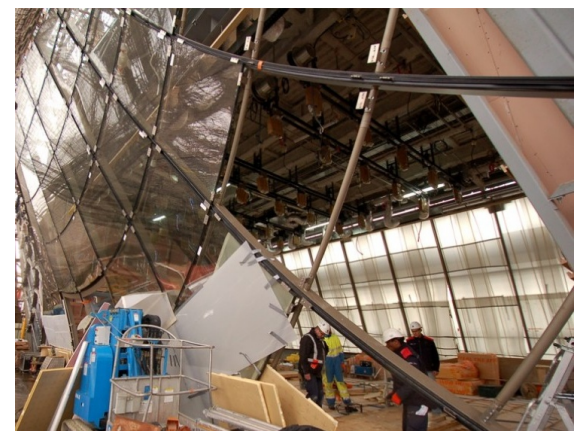

Figure 33: The Eiffel Tower Pavilions feature beams with rectangular cross-section whose side flanks are manufactured by bending. This makes them developable, and makes the entire beam arrangement a semidiscrete version of the support structure of Fig. 30, left (image: Evolute).

- Application: Shading systems. For certain applications, not the boundary meshes of a support structure are the main object of interest, but the collection of node axes (see Fig. 30, left). This has been demonstrated by Wang et al. [111] who use geometric support structures as shading systems. We refer to $\S 10$ and Figure 50.

- Support structures of hexagonal combinatorics. Triangle meshes with the "incircle-packing" property have a natural geometric support structure associated with them (see [88] and Figure 34): The vertices of the mesh are centers of balls which touch each other, and the common tangent planes carry the faces of the support structure. The aspect of packing led to a more general view of "cell packing structures" which are systematically discussed in a survey paper by Pottmann et al. [79].

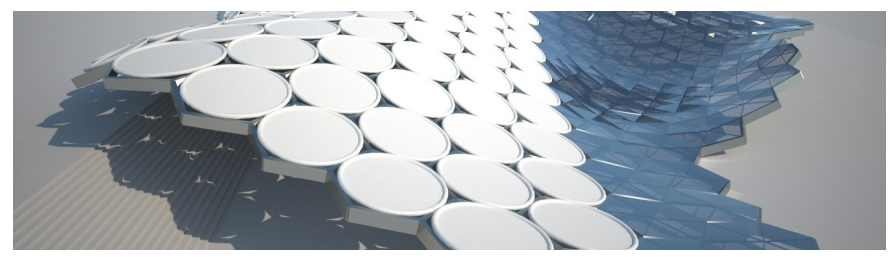

Figure 34: Hexagonal support structure. Here a triangle mesh (not shown) has the property that we can find spheres (shown only by their circular crosssections) centered in the vertices which touch each other. The common tangent planes of these spheres are the faces of a support structure (visible at boundary in front). This is part of a design created by H. Schmiedhofer for [88].

\subsection{Real projects involving torsion-free support structures.}

Large-scale freeform steel gridshells suffer from the problem of torsion in the nodes, where several steel beams meet. For triangular structures, this is basically unavoidable (cf. Figure 3). 
Quadrilateral structures have more degrees of freedom to remove or at least minimise node torsion. This fact was used for the enveloping quadrilateral structure of the Yas Marina Hotel in Abu Dhabi, which follows a geometric support structure in the sense defined here, see Figure 30. An even more literal realization of that kind of support structure is furnished by the roof of the Kogod courtyard, Smithsonian National Portrait Gallery, Washington DC, see Figure 51. A similar arrangement, but not based on quad mesh connectivity, is exhibited by the 2012 KREOD pavilions London, see Fig. 35.

\subsection{Support structures in the general sense}

The entire topic of structures which have a supporting function is, of course, a huge area in engineering which is not easily systematized. The following brief paragraphs on this topic focus on "interesting geometry" and are extremely selective.

We mention Vladimir Shukhov as a pioneer of modern structural engineering. His works engineered for the 1896 Nishny Novgorod fair (the hyperpoloid tower, his steel tensile structures, and the gridshell exhibition pavilion) were the first of their kind.

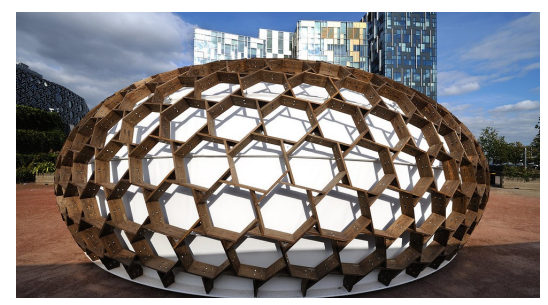

Figure 35: The hexagonal pattern exhibited by the KREOD pavilions is derived from the dual of a triangle mesh with the ball-packing property, leading to nice nodes and an even distribution of hexagons (image: KREOD).

As one example of a surprising development we mention tensegrity structures (consisting of elements of various kinds, e.g. cables allowing only tension, or struts only allowing compression), the term coined by Buckminster Fuller in 1960. A recent example is the 2009 Kurilpa Bridge, the world's largest tensegrity bridge. This topic is very rich also from the mathematical viewpoint, see e.g. [101] and [24].

A current trend is the use of free forms. This is seen e.g. in projects at a grand scale currently awaiting realization. We pick a random example, namely the Taiwan Tower by S. Fujimori (architect) and Bollinger and Grohmann (engineers, see [90]), with its "organic fiber" design. It is also seen in algorithmic tools such as the algorithmic approach to tree-like compression support structures for slabs by Lachauer and Block [61]. A significant number of physically realized freeform support structure are made from wood, where CNC machining can be easily employed. Examples are provided by the work of Shigeru Ban, such as the 2008 Centre Pompidou in Metz (France), cf. Fig. 36, or the 2008 Haesley Nine Bridges Golf Club. Intriguing wooden freeform structures can also be produced by layers of thin wooden plates, e.g. in the 2010 Rive Gauche Hermes Store in Paris, by Rena Dumas Architecture Interieure.

\subsection{Research directions}

- It would be very interesting to know what other structures besides the torsion-free support structures (in the narrow sense

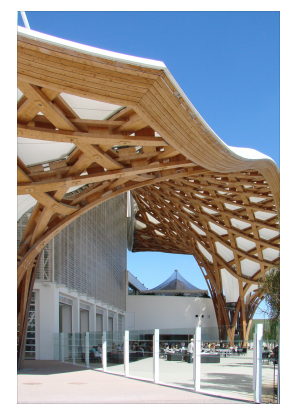

Figure 36: Wooden support structure, Centre Pompidou in Metz (France) by architect Shigeru Ban, and engineered by Arup in 2008. Production of the curved members was by CNC machining, with an estimated $50 \%$ of the raw material being chipped away during the process (photo: Annie Dalbéra).

of the word) have such a rich relation to geometry. Especially we would like to ask this question for fully 3D structures.

- In structures where nodes have valence 3, congruent nodes is a topic of current research, see also $§ 7$.

- We see inspiration from nature in many aspects, but biomimetic architecture is surely not yet fully explored, if the many successes of biomimetics in technology are any guide.

- It is possible to perfectly align prismatic beams (having the same cross-section) if they follow a mesh which has a parallel mesh at constant edge-edge distance [80]. It is known that these meshes constitute a discrete version of the Laguerre-isothermic surfaces, but it is an open problem to understand the variety of possible shapes of these meshes.

\section{Repetitive elements}

When paneling freeform surfaces, tolerances are often large enough to allow for repeating panel shapes (see §5). For structural elements like nodes, beams, and frames, however, the tolerances are often tighter and the geometry of these structures is often more complex than that of the outer skin. Therefore optimising freeform structures for repetitive elements is highly challenging and sometimes impossible. This complicates logistics and increases production cost, and is a typical feature of freeform shapes in architecture. The following paragraphs discusss some research on structures whose constituent parts exhibit repetition.

\subsection{Structures aiming at smoothness}

The aim of having as few differently shaped basic elements in a design motivated Singh and Schaefer [96] to optimize triangle meshes so that there is only a relatively small set of different faces up to some chosen tolerance. A similar approach to reduce the number of essentially different faces in (non-polyhedral) quad meshes has been taken by Fu et al. [36]. A common observation of these papers is that the goal of congruent faces works against mesh fairness. A similar behaviour is expected for the goal of having congruent node configurations in meshes with straight edges. However, giving up the straightness of edges and using circular arcs instead, one can achieve congruent and even regular nodes for triangular, quad and hex combinatorics (see [14] and Figure 37); covering a surface with families of congruent circular arcs is also possible [6]. 
Figure 37: For a proof of concept, the design surface of the Eindhoven Blob by M. Fuksas has been covered by circular arcs such that in each node 6 arcs meet at an angle of exactly 60 degrees between them. Moreover, each node is equipped with a virtual tangent plane tangent to all arcs emanating from that node.

\subsection{Non-smooth structures}

Element repetition for meshes is probably best achieved if one thinks of non-smoothness as a design element and intentionally plays with the rough surface, or with no surface at all. A strong indicator for the success of this line of thinking is work by Zimmer et al. [117] on triangle-based point folding structures. They represent freeform shapes by a non-smooth assembly of pyramidal panels of which only a few different kinds are used. Of a similar spirit is the work on representing freeform shapes with the plastic zometool construction set, offering only 9 different edges connecting nodes which allow to connect edges only in a finite number of angles [120, 119].

The previous examples concern the representation of arbitrary shapes with restricted means. We next discuss even more restricted geometric structures which no longer are capable of assuming all shapes. The so-called Lobel frames were introduced by the French architect Alain Lobel, who presented a large number of designs made from congruent equilateral triangles only. Lobel frames which are meshes, cf. Figure 38, appear to be non-smooth approximations of piecewise developable surfaces. This is in accordance with the fact that the "Lobel" mesh itself is developable as long as all vertices have degrees 6 and a total angle sum of $360^{\circ}$.

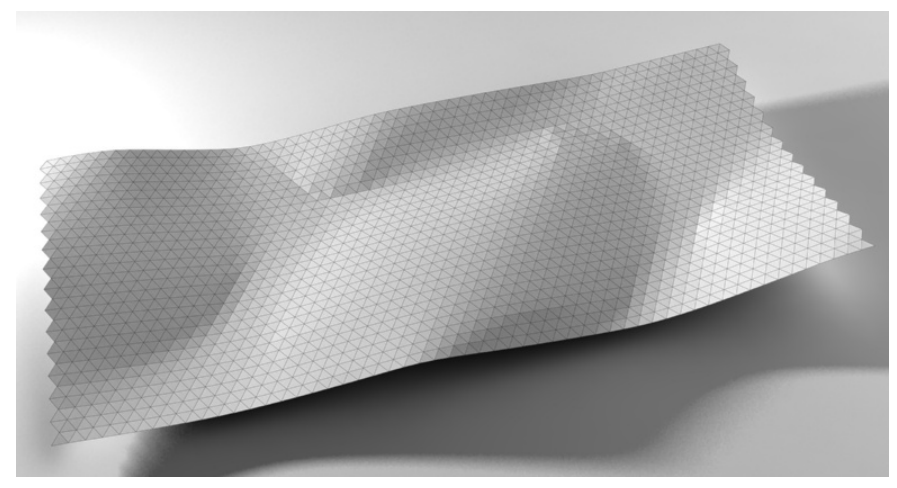

Figure 38: In a "Lobel" mesh consisting of equilateral triangles, the total angle sum at a valence 6 vertex is 360 degrees. This makes such meshes developable. This rendering, visualizing developability by tangential lighting, shows a Lobel mesh which approximates the design surface of the Cour Visconti courtyard in the Louvre. This approximation is far from accurate, which is consistent with the shape restrictions of developable surfaces, cf. [50, 47].

As to structures which contain repetition but which are not surfaces at all, we refer to the study of properties and optimization of honeycombs by [52], which are dual to "Lobel" meshes and which represent torsion-free support structures in the narrow sense discussed by $\S 6$, but with the additional property that all nodes are congruent. One can easily represent arbitrary shapes by honeycombs, but one cannot enforce the honeycomb's walls to be orthogonal to the reference surface unless it is developable. See Fig. 39 for an example.
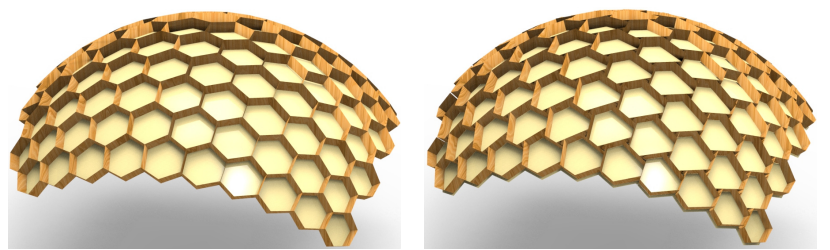

Figure 39: Honeycomb structures are defined as torsion-free support structures where all walls meet at $120^{\circ}$. These two examples have hex mesh combinatorics (left) and "reciprocal structure" combinatorics (right), cf. [52].

Discarding smoothness and replacing it by a different kind of regularity, Huard et al. [47] use a voxel-based method to represent shapes by planar panels guided by a small set of generators, see Figure 40. This does not only restrict panel shapes in the way one could say that "Lobel" meshes are generated by an equilateral triangle, but also restricts the positions of panels in space.

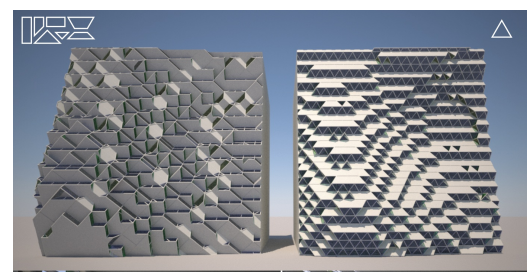

Figure 40: Planar panels which are repetitive in size, shape and position. Here the same reference surface has been represented by two different sets of generators, cf. [47].

\subsection{Real projects involving repetition}

In practice the problem of introducing repetition is circumvented rather than solved by not using true freeform structures at all. For example, complex shapes can be derived from basic geometries which admit symmetries, like tori for the Strasbourg train station (Fig. 16), or spheres in case of the Sydney Opera house.

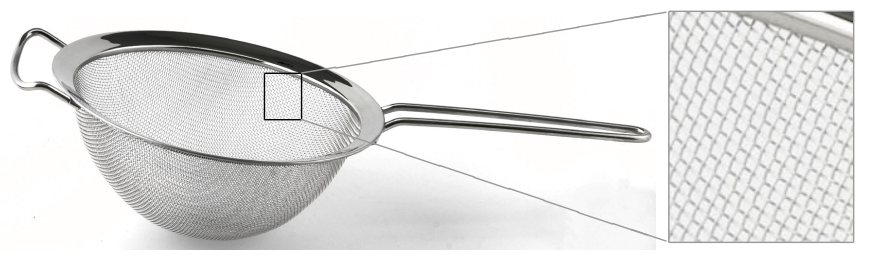

Figure 41: A tea strainer's domed shape can be flattended to produce a regular planar parallelogram lattice of wires. It represents a PQ mesh of constant edgelength, generated by parallel translating one polyline along another. Such shapes can approximate paraboloids of revolution, since paraboloids are also generated by parallel translating a parabola along another one.

Repetition in smaller elements includes meshes where all edges have the same length. This is a property claimed e.g. for the roof of the 1990 "Aquatoll Neckarsulm" swimming pool by engineers Schlaich Bergermann and Partners, and can be achieved by meshes which are generated by parallel translating one constant-edgelength polyline against another one, see Fig. 41. Another example of this principle is the 1974 "Mannheim Multihalle" where formfinding was based on a hanging 
chain model, see [40]. It is difficult to find examples where repetition has been enabled by optimization. Beaublanc Stadium in Limoges (in construction) was to be one: By a clustering algorithm it was tried to introduce repetition in the backing frames of the stadiums' triangular panels, itself consisting of a smaller triangle on the back of the panel. It turned out that tight tolerances prevented a useful level of repetition.

\subsection{Directions of further research}

- The digital design of "Lobel" frames deserves further study. Initial results have been achieved e.g. by [50].

- It is not yet clear how much repetition we can achieve if we give up the smoothness constraint, but still want to approximate arbitrary shapes.

- A related problem is the development of minimal sets of construction elements (like zometool) which offer sufficient flexibility for applications in architecture.

\section{Patterns}

Geometric patterns have fascinated mankind since ancient times. Artists had an excellent understanding of this subject and studied patterns and tilings thoroughly. This is especially true for the islamic world. In the context of freeform architecture, patterns can arise in many ways, including the arrangement of panels, the subconstruction, in additional functional layers such as shading systems or simply as textures. We here point to the few research contributions which look at these patterns not just as textures (which in computer graphics is a vast area in itself), but take an additional viewpoint, which then is mainly related to function and manufacturing.
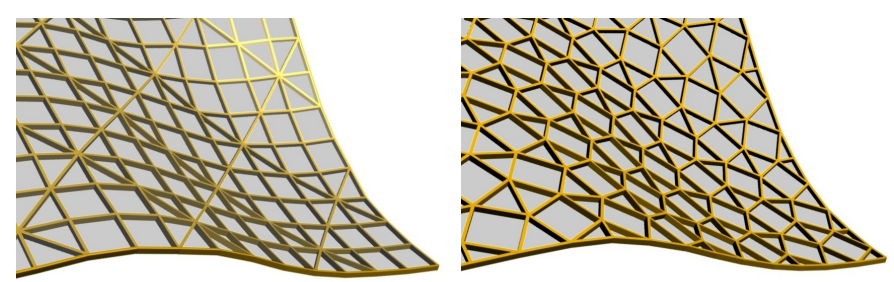

Figure 42: Different kinds of polyhedral patterns which exhibit triangular and flat quadrangular surfaces. In each case, the number of quadrangles is low enough not to hinder design freedom.

\subsection{Patterns from straight beams and flat panels}

Faces and edges of a polyhedral mesh may form a much more interesting geometric pattern than the simple ones coming from quad or triangle meshes of largely regular connectivity. The patterns can be a design element, but can also contribute to functional and manufacturing requirements. The hybrid mesh in the Cour Viscounti of the Louvre (covering the islamic art museum, see Figure 5) is a simple example. Some of the many further possible designs of hybrid polyhedral meshes covering the same design surface are illustrated in Fig. 42.

A very recent project exhibiting an ornamental polyhedral pattern is the Yas Mall gridshell in Abu Dhabi by Affan Innovative Structures (see Fig. 43). As this design exhibits mostly triangles, planarity of the few other faces is easy to achieve.
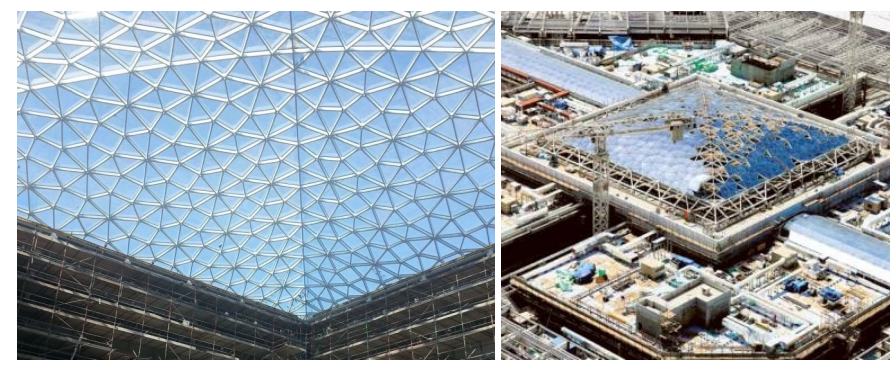

Figure 43: Yas Mall, Abu Dhabi: Gridshell with ornamental polyhedral pattern. Image at left: Stutzki Engineering Newsletter. Image at right, showing construction: Aldar.

Various remarkable patterns arise in connection with socalled circle packing meshes [88]: these are triangle meshes with the property that in-circles of adjacent faces touch each other on the common edge. The derived patterns appear in form of polyhedral meshes (see e.g. Fig. 44) or torsion free support structures.

If one considers a polyhedral mesh as an approximation of a smooth surface and therefore requires fairness properties of appropriate mesh polylines and of the Gaussian image, one is subject to restrictions imposed by differential geometry of surfaces. Examples for this are the relation between PQ meshes and conjugate parameterizations discussed in $\$ 2.3 .1$, or the non-convexity of planar faces in negatively curved regions shown in Figure 8. However, if one sacrifices fairness or interprets fairness in nonstandard ways, one may be able to overcome such restrictions. An example is seen in the tri-hex structure of Fig. 44, left, whose hexagons in negatively curved areas are convex, which is made possible by lack of fairness of the Gauss image mesh, cf. [88]. Another example is furnished by the quad mesh of Fig. 44, right, whose edges do not imitate a smooth network of curves, cf. [52].
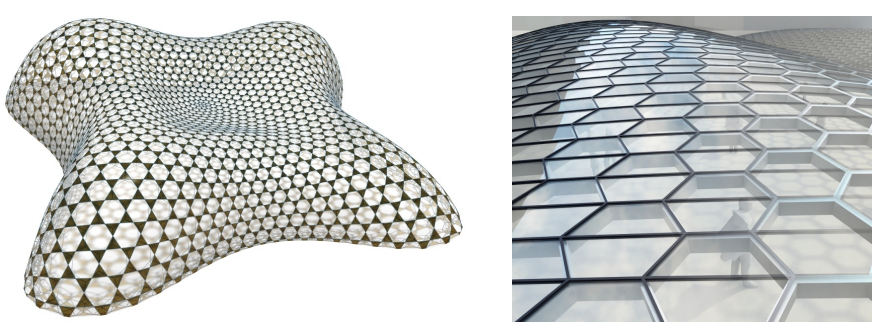

Figure 44: Polyhderal patterns whose smoothness is not expressible by smallness of differences of adjacent edges, and which have occurred as by-products of geometric investigations. Left: Tri-hex patterns derived from a circlepacking mesh such as shown by Figure 34. Right: Quad pattern derived from a "honeycomb" torsion-free support structure.

Song et al [98] recently developed an interactive design system for patterns from beams arranged in so-called reciprocal structures, exploiting the close relation of such structures to planar tilings from regular polygons.

\subsection{Patterns emerging from curved and long range elements}

Manufacturing constraints on architectural meshes may be imposed not only on faces or nodes, but also on long range ele- 
ments. For example, in a triangle mesh, one may impose special conditions on some or all of the dominating mesh polylines. Triangle meshes in which selected polylines are planar, circular or geodesic (i.e., shortest paths) have been investigated by Deng et al. [27]. This subject is related to the geometry of webs [8]: a triangle mesh of regular combinatorics is a discrete version of a hexagonal web.

In architecture, patterns of geodesics may arise naturally: timber support elements are preferrably aligned with geodesics [74] and cladding a surface with wooden panels which do not bend sideways implies that they have to follow geodesics (see [54, 78] and Fig. 13). The computational design of geodesic patterns benefits from the use of results from differential geometry, such as Jacobi fields and geodesic vector fields [78].

The two above-mentioned papers [78, 27] lay out a computational framework for the design of geodesic patterns of wooden elements which are manufacturable by bending (Fig. 45), as opposed to CNC milling (Fig. 36).

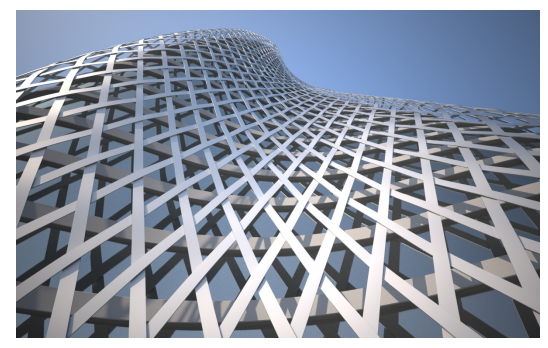

Figure 45: A 3-web of curves which are geodesic within tolerance, is the basis of this weaving design manufacturable by bending wooden panels. The geodesic property, if rigidly enforced, is a global property of a curve - see also Fig. 13.

\subsection{Brick patterns}

Architects Gramazio and Kohler use industrial robots to fabricate fascinating 3D structures, in some of which the emergence of patterns is a design element. We just mention here their seminal work on the robotic assembly of bricks to curved, nonstandardized walls [19, 7], which is an example of the fabrication-aware digital design mentioned in $\$ 1$. The robot approach has been pushed even further, to assembly by flying drones, see Figure 46 and [39].
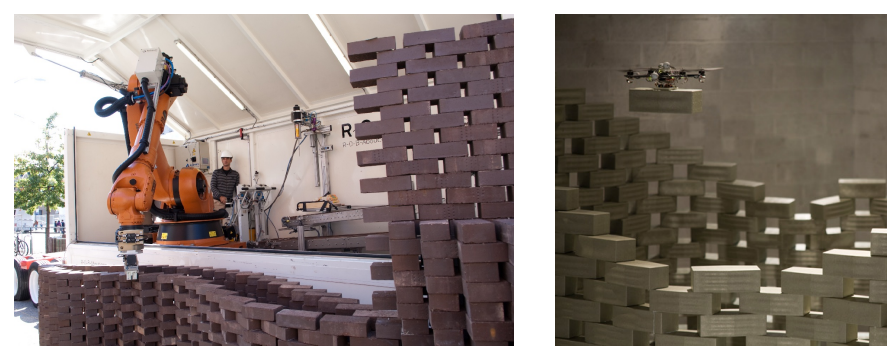

Figure 46: Robot-assembled and flight-assembled architecture. Left: Pike Loop project by Gramazio \& Kohler. A roboter is assembling a wiggled brick bond, cf. [7]. Right: Robot helicopters were programmed to stack bricks into a six metre-high tower at the FRAC Centre in Orléans, France, see [39].

\subsection{Real projects}

Today there exists a large variety of patterns on freeform structures, and an exhaustive treatment would exceed the scope of this paper. Patterns often define the visual impression of a building, and they may be formed by different means (seams of a paneling, a supporting structure,...). Already the projects discussed in the rest of this paper exhibit a large range of patterns, in many cases patterns from planar faces (triangular, quadrilateral, ... ). There are examples where a pattern exhibits mesh connectivity even if this is not apparent from visible vertices, edges and faces (Figure 47, left). Polygonal patterns containing random elements evolve if a surface is segmented by a set of cutting planes or curves (cf. the 2008 Beijing National Stadium, or the work by Toyo Ito shown in Figure 47, right).
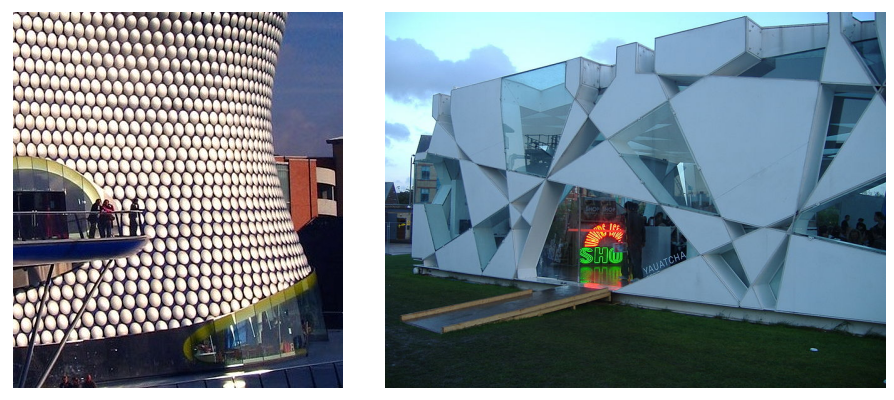

Figure 47: Patterns. Left: A pattern of circles covers Selfridges in Birmingham (2003, design by Future Systems). This pattern has regular triangular mesh connectivity, without vertices or edges being shown. Right: From the mathematical viewpoint, the 2002 temporary pavilion by Toyo Ito at the Serpentine Galleries (Hyde Park, London) has the combinatorics of a so-called arrangement of lines.

\subsection{Further research directions}

- It may be rewarding to systematically study patterns of flat panels in polyhedral surfaces which are capable of representing freeform shapes. For initial work in this see [52, 50].

- Maybe certain patterns, especially those which do not contain long dominating mesh polylines, have the potential to blend well with element repetition?

- There are many open but hard problems in the geometry of webs, which admittedly deserve interest mostly from a mathematical perspective, but may eventually form the basis of remarkable architectural designs. For example, a complete classification of all hexagonal webs from circles (in the plane or on the sphere) is still missing. It is easier to classify these circular webs if they are not in the plane or on the sphere, since then one can show that they must lie on so-called Darboux cyclides, whose circular webs have been recently classified [82].

\section{Statics-aware design}

\subsection{Self-supporting masonry}

Naturally, stability is of paramount importance in all architectural designs. However it is only recently that it can be taken into account during the design process in an automatic way. The complex nature of the question of stability and the involvement of many factors besides geometry makes statics-aware design feasible only in such situations where specifying the geometry already allows for statics analysis, and the designer is not held up by having to additionally specify structural elements 
in detail. Such a situation is present with shells and in particular with masonry. Here the work of Maxwell in the 19th century on reciprocal force diagrams [68] and Heyman's 1966 "safe theorem" model $[44,45,46]$ is the basis of recent work on finding discrete compressive force networks contained within the boundary of masonry structures, cf. [71, 2, 35]. Using such thrust networks for design is the work of P. Block and coauthors $[10,13,9,12,105,106]$. See Fig. 48 for an example.
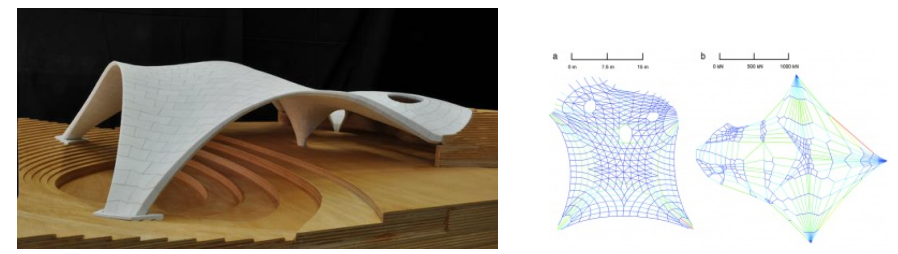

Figure 48: The Martin Luther King Jr. Park Stone Vault in Austin, Texas, represents freeform masonry which is stable once sliding of building blocks against each other is prevented. Left: model. Right: thrust network of compressive forces, and reciprocal force diagram (source: Block research group. ETH Zürich).

A different kind of approach is to represented structures by networks of rigid blocks [66], whose conditions on the structural feasibility were incorporated into procedural modeling of buildings [112]. Yet another method is to model structures as damped particle-spring systems ("dynamic relaxation" methods, cf. [57, 5]), mirroring the rich tradition in architecture of designing self-supporting surfaces using hanging chain or membrane models, for instance by Frei Otto and Antoni Gaudi. A new contribution is optimizing the topology of elastic grid shells by variational methods [42].

Returning to the thrust network method, its introduction by [9] was the motivation for further developments. One such developlment is to identify an auxiliary discrete surface generated from reciprocal force diagrams with a finite element discretization of the Airy potential which occurs in the continuous shell equations [35]. Vouga et al. [109] introduced the topic to graphics and geometric modeling, and interpreted thrust networks in the context of discrete differential geometry. This contribution invited follow-up work in geometric computing: [72] studies the tesselation of self-supporting surfaces into blocks, while $[38,63]$ deal with efficient design.
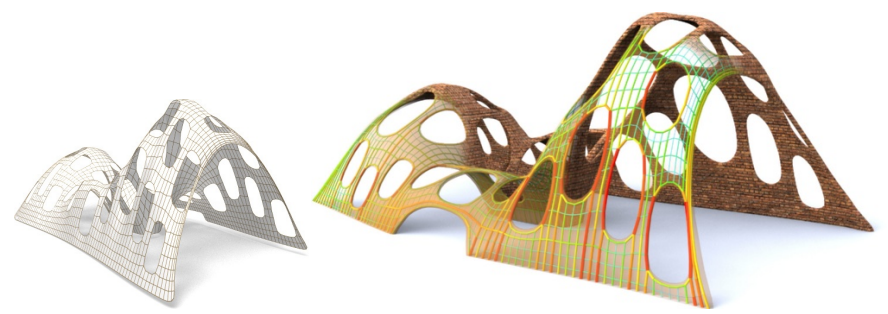

Figure 49: Optimizing surfaces for the self-supporting property. Left: Cutting holes in a self-supporting surface will destroy the self-supporting property. Right: Vouga et al. [109] show how to find the closest self-supporting surface. The image also shows the ficticious thrust network of forces which by Heyman's "safe theorem" guarantees stability.

Also the combination of statics-aware design with the topic of polyhedral surfaces (cf. §2) has been investigated: [86] dis- cuss statics-sensitive quad meshing with planar faces. Equilibrium forces, and the compressive nature of such forces, can however also be incorporated as additional side conditions in the modeling of polyhedral meshes (which is done by [100], see $§ 11)$ ).

\subsection{Real projects}

Methods of statics-aware design have mostly been applied to masonry. Freeform masonry has been in existence for many centuries. Impressive width/span ratios have been achieved by gothic vaults, e.g. by the chapel of King's College in Cambridge. As to real projects which are actually based on the methods discussed in this chaper, we have already mentioned the stone vault to be built in the Martin Luther King Jr. Park in Austin, Texas (Figure 48). The structural vaults of a new museum at Mapungubwe, South Africa, are conceptually similar, and so was a smaller temporary installation of a freeform Catalan vault on the campus of ETH Zürich (the term Catalan vault refers to the technique of laying bricks over a wooden form).

\subsection{Open problems and research directions}

The area of statics-aware design has been explored only to a very small degree, which can be seen already from the very specific and narrow nature of the topics discussed in this section. Open problems include:

- Implement a system for form-findig which combines statics, manufacturing and further aspects like shading and lighting.

- Intuitively, some shapes are more stable than others. Investigate which of the possible ways of exactifying this property is algorithmically accessible, especially for finding "most stable" shapes.

- Motivated by the Catalan vault technique, we are interested in the the question of minimal scaffolding for structures which are eventually self-supporting once they are complete, but do not have this property during assembly. [28] has recent results in this direction.

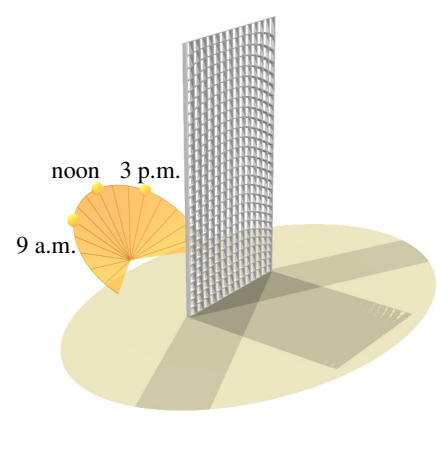

Figure 51: The roof of the Kogod courtyard in the Smithsonian Portrait Gallery by Foster and partners physically realizes a torsionfree support structure. It is optimized also for shading (image: Foster and partners).
Figure 50: Geometric support structures can be employed as shading systems, for freeform skins as well as for conventional flat facades. When optimizing for optimal blocking of light, the connecivtity of the shading system is part of the solution, see [111].

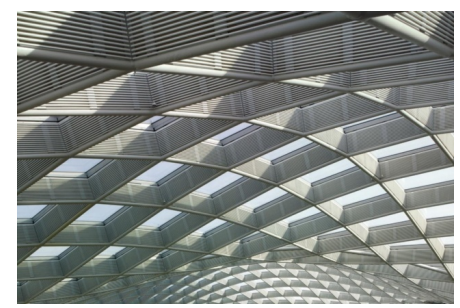




\section{Shading, lighting and other functional aspects}

\subsection{Shading and lighting systems}

The distribution of light within a building is of great importance, but so far has received little attention from the Architectural Geometry community. Several of the geometric structures discussed in this paper are relevant for shading, e.g. torsionfree support structures (see Figure 51), which can be optimized for shading effect. A more general treatment of this topic has been based on interpreting them as discrete line congruences and using basic insights from line geometry for the corresponding optimization $[111,51]$, see Fig. 50. Extensions to structures which are not only quad-based have been briefly addressed by [79], but there is a lot of room for improvement and alternative realizations involving freeform geometry.

\subsection{Kinematic flexibility in structures}

Dynamic elements are very attractive in architecture. For some purposes, in particular shading and lighting, the transformation of one geometric shape to another is even necessary for function - static structures are limited in their potential to accurately control daylight's access to a building. Research in this area includes [41], where existing software tools are combined to achieve light control by origami lightweight structures. Kinetic shading systems have actually been realized, e.g. for the 2010 "Aldar Central Market" in Abu Dhabi, using a system developed by a C. Hoberman and Buro Happold joint venture.

A very interesting direction of research is on designs which are able to change their size (such as C. Hoberman's spheres, see below) or their shape. There are, however, not many contributions to this question. T. Tachi [99] studies foldable structures inspired by origami. In $\S 4.4$ we have already mentioned Barton et al. [6] who presented tranformable designs from circular arcs joined smoothly, cf. Figure 26.
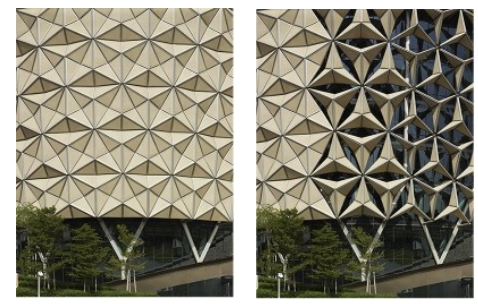

Figure 52: The Al-Bahar office towers in Abu Dhabi by Aedas architects feature an automated dynamic external shading system inspired by traditional islamic motifs.

\subsection{Real projects}

Shading structures have of course been built for a long time, and effective techniques evolved by way of practice and experiment. We only mention that various references on the use of geometry and light in art and architecture can be found in [59], and for a recent example we point to the large Dome covering the new Louvre Abu Dhabi, which consists of several layers of metal cladding optimised for both shading and casting visible rays of light onto the "micro-city" covered by the dome [60].

For real projects involving dynamic elements, we refer to the Al-Bahar Office towers in Abu Dhabi, see Figure 52, and solutions by Hoberman Associates. C. Hoberman is widely

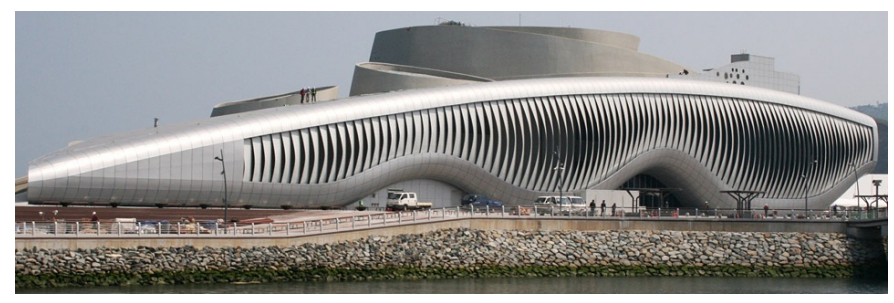

Figure 53: A kinetic facade based on bionic principles by Knippers Helbig, Stuttgart, is part of the 2012 One Ocean thematic pavilion by Soma Architecture, which has been built in Yeosu, South Korea.

known especially for his concept of transformable design. These are animated structures which change over time. The most famous is the well-known Hoberman sphere, where a sphere changes its size due to a complicated folding mechanism. Also hyperbolic surfaces like a transformable helicoid have been realized in this way.

Biomimetics research by J. Knippers and S. Schleicher at the Institute of Building Structures and Structural Design, University of Stuttgart takes inspiration from plant movement to develop novel bending and folding structures for various applications, including adaptive shading systems. Their product Flectofin is inspired by the pollination mechanism of strelitzia reginae - see Figure 53.

Striking designs do not need to employ novel mechanisms: the eye-shaped L'Hemisfèric building by S. Calatrava employs a series of folding-door mechanisms to open gigantic lids.

\subsection{Further research directions}

The areas discussed in this section represent a broad field of research possibilities.

- Shading and lighting based on flexible structures is widely open for future research, and the same is true for shape changing transformable design.

- In general, kinetic / transformable / flexible structures in architecture constitute a promising but difficult research direction. Challenges include the necessity of dynamics analysis, in addition to statics.

- Inspiration from nature has a great protential, not only for kinetic designs.

\section{Interactive design systems and design exploration}

Technical papers about the geometric problems related with freeform architecture sometimes create the impression that those problems are solved by an optimization process which has a unique solution. Such a situation of course would be at odds with the creative processes instrumental to both design and architecture. Obeying constraints is of course nothing new to designers, and architects are well accustomed to statics and properties of materials. However, within the context of architectural geometry there are side-conditions which are of a complex geometric nature and which are not easily understood without computational assistance. As we have seen above, such constraints 
may come from fabrication, statics, space requirements, lighting and other sources.

As to the manifold and varying nature of design exploration, the reader is referred to [56]. This section does not aim for that kind of generality and discusses design exploration only in so far as the above-mentioned constraints are concerned: In order for an artist to make full use of all degrees of freedom (subject to constraints), it is necessary to find efficient algorithms for exploring the design space, and to investigate design variations. In this way a designer can finally select that design which best matches the original intent.

Design variations can be actively generated by using various types of editing tools, which are most frequently handlebased. For advanced tasks it is crucial that the editing tool goes beyond standard mesh editing and produces results which fulfill the constraints. Unfortunately, commercially available software does not provide such tools at the time of writing. Contributions to this problem are provided by [25] (achieving speed by exploiting the GPU, but disregarding statics) and [100] (achieving speed by a clever choice of variables and by not attempting optimization).

Another way of exploring the design space is to let the system suggest some variations or expose the user in an appropriate way to the space of admissible designs. One solution for this problem has been proposed by Yang et al. $[115,26]$. They consider meshes and view the collection of the $N$ vertices which are allowed to change as a point in $3 N$-dimensional space. In that space, the constraints define a certain set to be explored. An appropriate collection of quality functions defines those part of the constraint variety which are useful (e.g. aesthetically pleasing) designs. The method of Yang et al. works by approximation and does not allow for a change in combinatorics. For the special case of polyhedral meshes, Poranne et al. [75] devised a method for exploring the linear subspaces in the constraint variety.

Another approach to exploring the design space is to parametrize it with variables other than the vertices, so that their manipulation preserves the necessary properties by design. In order to preserve planarity of faces in a deformation, [107] used per-face affine maps, and [108] extended them to projective maps. Using these maps as variables, it is possible to perform some deformation, interpolation and other editing operations, obeying planarity constraints.

\section{Research directions}

- We need a better understanding of shape spaces (constraint varieties). They depend on the used variables (coordinates). Exploration based on simple submanifolds (in extension of the linear subspaces in the space of polyhedral surfaces) could be interesting, especially from a geometric perspective.

- So far design space exploration has been largely based on local approximations of the constraint variety. More global exploration strategies would be highly welcome.

- Freeform skins have to be studied in connection with requirements on the interior of a building.

- There seems to be a lot of room for research on computational design systems dealing with standard architecture and considering additional aspects such as energy efficiency (e.g. via the optimized control of sun light entering the building).

- In order to respect aspects of function and fabrication, one has to combine geometric design with simulations. The latter have to be simplified so as to capture the essential effects and allow for interactive design. Such design tools are not limited to architecture and are expected to become popular in many other application areas. An example of such a system for the case of furniture design has been presented by Umetani et al. [104].

\section{Conclusion}

Our aim was to compile an overview of recent research around freeform architecture in an effort to identify core tasks and results, illustrate the discussion by real world examples and to outline some of the many directions for future research. Partially those go well beyond architecture, which probably constitutes the most important message of this survey: Next generation geometric modeling systems should be much easier to use and contribute to a shorter product development cycle. One way of getting closer to this goal is to integrate simple simulations and constraints around key aspects of function, fabrication and usability into shape modeling systems. As remarked by a reviewer of this paper, there are particular challenges in implementation: All these tools should be made available in a way which supports and broadens the design process rather than having a restrictive effect by providing ready-made solutions.

\section{Acknowledgments}

This research was supported by the Austrian Science Fund (FWF) through grant P23735-N13 and by the DFG-Collaborative Research Center, TRR 109 Discretization in Geometry and Dynamics, through FWF grants I705-N26 and I706-N26. The authors would like to thank Philippe Bompas and Jacques Raynaud for helpful discussions. We are very grateful to the anonymous reviewers for their extensive and helpful comments.

\section{References}

[1] Almegaard, H., Bagger, A., Gravesen, J., Jüttler, B., \& Š́r, Z. (2007). Surfaces with piecewise linear support functions over spherical triangulations. In M. Sabin, \& J. Winkler (Eds.), Mathematics of Surfaces XII (pp. 42-63). Springer.

[2] Andreu, A., Gil, L., \& Roca, P. (2007). Computational analysis of masonry structures with a funicular model. J. Engrg. Mech., 133, 473-480.

[3] Aumann, G. (2004). Degree elevation and developable Bézier surfaces. Computer Aided Geom. Design, 21, 661-670.

[4] Baldassini, N., Leduc, N., \& Schiftner, A. (2013). Construction aware design of curved glass facades: The Eiffel Tower Pavilions. In Glass Performance Days Finland (Conference Proceedings) (pp. 406-10).

[5] Barnes, M. R. (2009). Form finding and analysis of tension structures by dynamic relaxation. Int. J. Space Struct., 14(2), 89-104.

[6] Bartoň, M., Shi, L., Pottmann, H., Kilian, M., \& Wallner, J. (2013). Circular arc snakes and kinematic surface generation. Comput. Graph. Forum, 32, 1-10. Proc. Eurographics.

[7] Bärtschi, R., Knauss, M., Bonwetsch, T., Gramazio, F., \& Kohler, M. (2010). Wiggled brick bond. In C. Ceccato et al. (Eds.), Advances in Architectural Geometry 2010 (pp. 137-47). Springer.

[8] Blaschke, W., \& Bol, G. (1938). Geometrie der Gewebe. Springer. 
[9] Block, P. (2009). Thrust Network Analysis: Exploring Three-dimensional Equilibrium. Ph.D. thesis M.I.T.

[10] Block, P., Ciblac, T., \& Ochsendorf, J. (2006). Real-time limit analysis of vaulted masonry buildings. Computers and Structures, 84, 18411852.

[11] Block, P., Knippers, J., Mitra, N., \& Wang, W. (Eds.) (2014). Advances in Architectural Geometry 2014. Springer.

[12] Block, P., \& Lachauer, L. (2011). Closest-fit, compression-only solutions for free form shells. In Proc. IABSE - IASS Symposium.

[13] Block, P., \& Ochsendorf, J. (2007). Thrust network analysis: A new methodology for three-dimensional equilibrium. J. Int. Assoc. Shell and Spatial Structures, 48(3), 167-173.

[14] Bo, P., Pottmann, H., Kilian, M., Wang, W., \& Wallner, J. (2011). Circular arc structures. ACM Trans. Graph., 30, \#101,1-11.

[15] Bobenko, A., Pottmann, H., \& Wallner, J. (2010). A curvature theory for discrete surfaces based on mesh parallelity. Math. Annalen, 348, 1-24.

[16] Bobenko, A., \& Suris, Yu. (2008). Discrete differential geometry: Integrable Structure. American Math. Soc.

[17] Bobenko, A. I., \& Huhnen-Venedey, E. (2012). Curvature line parametrized surfaces and orthogonal coordinate systems. Discretization with Dupin cyclides. Geom. Dedicata, 159, 207-237.

[18] Bommes, D., Campen, M., Ebke, H.-C., Alliez, P., \& Kobbelt, L. (2013). Integer-grid maps for reliable quad meshing. ACM Trans. Graph., 32(4), \#98,1-12. Proc. SIGGRAPH.

[19] Bonwetsch, T., Gramazio, F., \& Kohler, M. (2007). Digitally fabricating non-standardized brick walls. In M. Sharp (Ed.), ManuBuild (1st International Conference) (pp. 191-6). London: CIRIA.

[20] Bouaziz, S., Schwartzburg, Y., Weise, T., \& Pauly, M. (2012). Shaping discrete geometry with projections. Computer Grap. Forum, 31, 16571667. Proc. SGP.

[21] Ceccato, C., Hesselgren, L., Pauly, M., Pottmann, H., \& Wallner, J. (Eds.) (2010). Advances in Architectural Geometry 2010. Springer.

[22] Chu, C. H., \& Séquin, C. (2002). Developable Bézier patches: properties and design. Comp. Aided Design, 34, 511-528.

[23] Cohen-Steiner, D., Alliez, P., \& Desbrun, M. (2004). Variational shape approximation. ACM Trans. Graphics, 23(3), 905-914. Proc. SIGGRAPH.

[24] Connelly, R. (2013). Tensegrities and global rigidity. In M. Senechal (Ed.), Shaping Space (pp. 267-78). Springer.

[25] Deng, B., Bouaziz, S., Deuss, M., Kaspar, A., Schwartzburg, Y., \& Pauly, M. (2015). Interactive design exploration for constrained meshes. Computer-Aided Design, 61, 13-23.

[26] Deng, B., Bouaziz, S., Deuss, M., Zhang, J., Schwartzburg, Y., \& Pauly, M. (2013). Exploring local modifications for constrained meshes. Comput. Graph. Forum, 32(2), 11-20. Proc. Eurographics.

[27] Deng, B., Pottmann, H., \& Wallner, J. (2011). Functional webs for freeform architecture. Comput. Graph. Forum, 30, 1369-1378. Proc. SGP.

[28] Deuss, M., Panozzo, D., Whiting, E., Liu, Y., Block, P., SorkineHornung, O., \& Pauly, M. (2014). Assembling self-supporting structures. ACM Trans. Graphics, 33. Proc. SIGGRAPH Asia, to appear.

[29] Ebke, H.-C., Bommes, D., Campen, M., \& Kobbelt, L. (2013). QEx: Robust quad mesh extraction. ACM Trans. Graph., 32(6), \#168,1-10.

[30] Eigensatz, M., Kilian, M., Schiftner, A., Mitra, N., Pottmann, H., \& Pauly, M. (2010). Paneling architectural freeform surfaces. ACM Trans. Graph., 29(4), \#45,1-10. Proc. SIGGRAPH.

[31] Eigensatz, M., Schiftner, A., Deuss, M., Kilian, M., Mitra, N., Pottmann, H., \& Pauly, M. (2010). Case studies in cost-optimized paneling of architectural freeform surfaces. In C. Ceccato et al. (Eds.), Advances in Architectural Geometry 2010 (pp. 49-72). Springer

[32] Flöry, S. (2010). Constrained Matching of Point Clouds and Surfaces. $\mathrm{Ph} . \mathrm{D}$. thesis TU Wien. 四

[33] Flöry, S., Nagai, Y., Isvoranu, F., Pottmann, H., \& Wallner, J. (2012). Ruled free forms. In L. Hesselgren et al. (Eds.), Advances in Architectural Geometry 2012 (pp. 57-66). Springer.

[34] Flöry, S., \& Pottmann, H. (2010). Ruled surfaces for rationalization and design in architecture. In A. Sprecher et al. (Eds.), LIFE in:formation. On Responsive Information and Variations in Architecture (pp. 103-9). Proc. ACADIA.

[35] Fraternali, F. (2010). A thrust network approach to the equilibrium problem of unreinforced masonry vaults via polyhedral stress functions. Mechanics Res. Comm., 37(2), 198-204.
[36] Fu, C.-W., Lai, C.-F., He, Y., \& Cohen-Or, D. (2010). K-set tilable surfaces. ACM Trans. Graph., 29, \#44,1-6. Proc. SIGGRAPH.

[37] Glymph, J., Shelden, D., Ceccato, C., Mussel, J., \& Schober, H. (2004). A parametric strategy for free-form glass structures using quadrilateral planar facets. Automation in Construction, 13(2), 187-202.

[38] de Goes, F., Alliez, P., Owhadi, H., \& Desbrun, M. (2013). On the equilibrium of simplicial masonry structures. ACM Trans. Graph., 32(4), \#93,1-10. Proc. SIGGRAPH.

[39] Gramazio, F., Kohler, M., \& d'Andrea, R. (2013). Flight Asembled Architecture. Editions Hyx. Exhibition catalogue.

[40] Happold, E., \& Liddell, W. I. (1975). Timber lattice roof for the Mannheim Bundesgartenschau. The Structural Engineer, 53(3), 99-135.

[41] Heinzelmann, F. (2009). Origami lightweight structure and daylighting modulation. In C. Gengnagel (Ed.), Proc. Design Modelling Symposium Berlin 2009 (pp. 207-17). UdK Berlin.

[42] Hernandez, E. L., Sechelmann, S., Rörig, T., \& Gengnagl, C. (2012). Topology optimisation of regular and irregular elastic gridshells by means of a non-linear variational method. In L. Hesselgren et al. (Eds.), Advances in Architectural Geometry 2012 (pp. 147-60). Springer

[43] Hesselgren, L., Sharma, S., Wallner, J., Baldassini, N., Bompas, P., \& Raynaud, J. (Eds.) (2013). Advances in Architectural Geometry 2012. Springer.

[44] Heyman, J. (1966). The stone skeleton. Int. J. Solids Structures, 2, 249-279.

[45] Heyman, J. (1995). The Stone Skeleton: Structural Engineering of Masonry Architecture. Cambridge Univ. Press.

[46] Heyman, J. (1998). Structural Analysis: A Historical Approach. Cambridge Univ. Press.

[47] Huard, M., Bompas, P., \& Eigensatz, M. (2014). Planar panelization with extreme repetition. In P. Block et al. (Eds.), Advances in Architectural Geometry 2014. Springer.

[48] Huhnen-Venedey, E., \& Rörig, T. (2014). Discretization of asymptotic line parametrizations using hyperboloid surface patches. Geometriae Dedicata, 168, 265-289.

[49] Huhnen-Venedey, E., \& Schief, W. K. (2014). On Weingarten transformations of hyperbolic nets. Int. Math. Res. Notices, . To appear.

[50] Jiang, C., Tang, C., Tomičić, M., Wallner, J., \& Pottmann, H. (2014). Interactive modeling of architectural freeform structures - combining geometry with fabrication and statics. In P. Block et al. (Eds.), Advances in Architectural Geometry 2014. Springer.

[51] Jiang, C., Wang, J., Bompas, P., Pottmann, H., \& Wallner, J. (2013). Freeform shading and lighting systems from planar quads. In C. Gengnagel et al. (Eds.), Rethinking Prototyping (pp. 335-46). Berlin: epubli $\mathrm{GmbH}$. Proc. Design Modelling Symposium.

[52] Jiang, C., Wang, J., Wallner, J., \& Pottmann, H. (2014). Freeform honeycomb structures. Comput. Graph. Forum, 33(5), 185-194. Proc. SGP.

[53] Käferböck, F., \& Pottmann, H. (2013). Smooth surfaces from bilinear patches: discrete affine minimal surfaces. Computer Aided Geom. Design, 30, 476-489.

[54] Kahlert, J., Olson, M., \& Zhang, H. (2011). Width-bounded geodesic strips for surface tiling. The Visual Computer, 27(1), 45-56.

[55] Kalogerakis, E., Hertzmann, A., \& Singh, K. (2010). Learning 3D mesh segmentation and labeling. ACM Trans. Graph., 29, \#102,1-12. Proc. SIGGRAPH.

[56] Kilian, A. (2006). Design exploration through bidirectional modeling of constraints. Ph.D. thesis M.I.T.

[57] Kilian, A., \& Ochsendorf, J. (2005). Particle-spring systems for structural form finding. J. Int. Assoc. Shell Spatial Struct., 46, 77-84.

[58] Kilian, M., Flöry, S., Chen, Z., Mitra, N., Sheffer, A., \& Pottmann, H. (2008). Curved folding. ACM Trans. Graph., 27(3), \#75,1-9. Proc. SIGGRAPH

[59] Kiser, T., Eigensatz, M., Nguyen, M. M., Bompas, P., \& Pauly, M. (2012). Architectural caustics - controlling light with geometry. In L. Hesselgren et al. (Eds.), Advances in Architectural Geometry 2012 (pp. 91-106). Springer.

[60] Koren, B. S. (2010). Louvre Abu Dhabi 1/33 - fabrication of a largescale physical light-test model. In C. Ceccato et al. (Eds.), Advances in Architectural Geometry 2010 (pp. 163-74). Springer.

[61] Lachauer, L., \& Block, P. (2012). Compression support structures for slabs. In L. Hesselgren et al. (Eds.), Advances in Architectural Geometry 2012 (pp. 135-46). Springer. 
[62] Li, Y., Zhang, E., Kobayashi, Y., \& Wonka, P. (2010). Editing operations for irregular vertices in triangle meshes. ACM Trans. Graph., 29(6), \#153,1-12. Proc. SIGGRAPH Asia.

[63] Liu, Y., Pan, H., Snyder, J., Wang, W., \& Guo, B. (2013). Computing self-supporting surfaces by regular triangulation. ACM Trans. Graph., 32(4), \#92,1-10. Proc. SIGGRAPH.

[64] Liu, Y., Pottmann, H., Wallner, J., Yang, Y.-L., \& Wang, W. (2006). Geometric modeling with conical meshes and developable surfaces. ACM Trans. Graph., 25(3), 681-689. Proc. SIGGRAPH.

[65] Liu, Y., Xu, W., Wang, J., Zhu, L., Guo, B., Chen, F., \& Wang, G. (2011). General planar quadrilateral mesh design using conjugate direction field. ACM Trans. Graph., 30, \#140, 1-10. Proc. SIGGRAPH Asia.

[66] Livesley, R. K. (1992). A computational model for the limit analysis of three-dimensional masonry structures. Meccanica, 27, 161-172.

[67] Martin, R. R., de Pont, J., \& Sharrock, T. J. (1986). Cyclide surfaces in computer aided design. In J. A. Gregory (Ed.), The mathematics of surfaces (pp. 253-68). Clarendon Press.

[68] Maxwell, J. (1864). On reciprocal diagrams and diagrams of forces. Philosophical Magazine, 4(27), 250-261.

[69] Meredith, N., \& Kotronis, J. (2012). Self-detailing and self-documenting systems for wood fabrication: The Burj Khalifa. In L. Hesselgren et al. (Eds.), Advances in Architectural Geometry 2012 (pp. 185-98). Springer.

[70] Milson, R. (2001). An overview of Lie's line-sphere correspondence. Contemp. Math., 285, 1-10.

[71] O'Dwyer, D. (1998). Funicular analysis of masonry vaults. Computers and Structures, 73, 187-197.

[72] Panozzo, D., Block, P., \& Sorkine-Hornung, O. (2013). Designing unreinforced masonry models. ACM Trans. Graph., 32(4), \#91,1-12. Proc. SIGGRAPH.

[73] Peng, C.-H., Barton, M., Jiang, C., \& Wonka, P. (2014). Exploring quadrangulations. ACM Trans. Graph., 33, \#12,1-13.

[74] Pirazzi, C., \& Weinand, Y. (2006). Geodesic lines on free-form surfaces: optimized grids for timber rib shells. In 9th World Conference on Timber Engineering (pp. 72-9).

[75] Poranne, R., Chen, R., \& Gotsman, C. (2013). On linear spaces of polyhedral meshes. Preprint arXiv:1303.4110.

[76] Poranne, R., Ovreiu, E., \& Gotsman, C. (2013). Interactive planarization and optimization of 3D meshes. Comput. Graph. Forum, 32(1), 152163.

[77] Pottmann, H., Asperl, A., Hofer, M., \& Kilian, A. (2007). Architectural Geometry. Bentley Institute Press.

[78] Pottmann, H., Huang, Q., Deng, B., Schiftner, A., Kilian, M., Guibas, L., \& Wallner, J. (2010). Geodesic patterns. ACM Trans. Graph., 29(4), \#43,1-10. Proc. SIGGRAPH.

[79] Pottmann, H., Jiang, C., Höbinger, M., Wang, J., Bompas, P., \& Wallner, J. (2015). Cell packing structures. Computer-Aided Design, 50, 70-83. To appear.

[80] Pottmann, H., Liu, Y., Wallner, J., Bobenko, A., \& Wang, W. (2007). Geometry of multi-layer freeform structures for architecture. ACM Trans. Graph., 26, \#65,1-11. Proc. SIGGRAPH.

[81] Pottmann, H., Schiftner, A., Bo, P., Schmiedhofer, H., Wang, W., Baldassini, N., \& Wallner, J. (2008). Freeform surfaces from single curved panels. ACM Trans. Graph., 27(3), \#76,1-10. Proc. SIGGRAPH.

[82] Pottmann, H., Shi, L., \& Skopenkov, M. (2012). Darboux cyclides and webs from circles. Computer Aided Geom. Design, 29, 77-97.

[83] Pottmann, H., \& Wallner, J. (2001). Computational Line Geometry. Springer.

[84] Sauer, R. (1970). Differenzengeometrie. Springer.

[85] Schiftner, A. (2007). Planar quad meshes from relative principal curvature lines. Master's thesis TU Wien. 雷.

[86] Schiftner, A., \& Balzer, J. (2010). Statics-sensitive layout of planar quadrilateral meshes. In C. Ceccato et al. (Eds.), Advances in Architectural Geometry 2010 (pp. 221-36). Springer.

[87] Schiftner, A., Eigensatz, M., Kilian, M., \& Chinzi, G. (2013). Large scale double curved glass facades made feasible - the Arena Corinthians west facade. In Glass Performance Days Finland (Conference Proceedings) (pp. $494-8$ ).

[88] Schiftner, A., Höbinger, M., Wallner, J., \& Pottmann, H. (2009). Packing circles and spheres on surfaces. ACM Trans. Graph., 28(5), \#139,1-8. Proc. SIGGRAPH Asia.
[89] Schiftner, A., Leduc, N., Bompas, P., Baldassini, N., \& Eigensatz, M. (2012). Architectural geometry from research to practice - the Eiffel Tower Pavilions. In L. Hesselgren et al. (Eds.), Advances in Architectural Geometry 2012 (pp. 213-28). Springer.

[90] Schinegger, K., Rutzinger, S., Hofmann, A., \& Hauer, D. (2012). Multiple nature - Taiwan tower. In L. Hesselgren et al. (Eds.), Advances in Architectural Geometry 2012 (pp. 229-36). Springer.

[91] Schneider, M., \& Mehrtens, P. (2012). Cladding freeform surfaces with curved metal panels - a complete digital production chain. In L. Hesselgren et al. (Eds.), Advances in Architectural Geometry 2012 (pp. $237-$ 42). Springer.

[92] Schober, H. (2003). Freeform glass structures. In J. Vitkala (Ed.), Glass Processing Days (pp. 46-50). Tamglass.

[93] Sechelmann, S., Rörig, T., \& Bobenko, A. (2012). Quasiisothermic mesh layout. In L. Hesselgren et al. (Eds.), Advances in Architectural Geometry 2012 (pp. 243-58). Springer.

[94] Shelden, D. (2002). Digital surface representation and the constructibility of Gehry's architecture. Ph.D. thesis M.I.T. 四.

[95] Shi, L., Wang, J., \& Pottmann, H. (2014). Smooth surfaces from rational bilinear patches. Computer Aided Geom. Design, 31, 1-12.

[96] Singh, M., \& Schaefer, S. (2010). Triangle surfaces with discrete equivalence classes. ACM Trans. Graph., 29, \#46,1-7. Proc. SIGGRAPH.

[97] Solomon, J., Vouga, E., Wardetzky, M., \& Grinspun, E. (2012). Flexible developable surfaces. Comput. Graph. Forum, 31, 1567-1576. Proc. SGP.

[98] Song, P., Fu, C.-W., Goswami, P., Zheng, J., Mitra, N., \& Cohen-Or, D. (2013). Reciprocal frame structures made easy. ACM Trans. Graph., 32(4), \#94,1-10. Proc. SIGGRAPH.

[99] Tachi, T. (2010). Freeform rigid-foldable structure using bidirectionally flat-foldable planar quadrilateral mesh. In C. Ceccato et al. (Eds.), Advances in Architectural Geometry 2010 (pp. 87-102). Springer.

[100] Tang, C., Sun, X., Gomes, A., Wallner, J., \& Pottmann, H. (2014). Formfinding with polyhedral meshes made simple. ACM Trans. Graphics, 33(4). Proc. SIGGRAPPH.

[101] Tibert, A. G., \& Pellegrino, S. (2003). Form-finding methods for tensegrity structures. Int. J. Space Structures, 18(4), 209-223.

[102] Troche, C. (2008). Planar hexagonal meshes by tangent plane intersection. In Advances in Architectural Geometry (pp. 57-60). TU Wien.

[103] Troche, C. (2009). Planar hexagonal tesselation of freeform surfaces manufacturing solutions and design potentials. In C. Gengnagel (Ed.), Proc. Design Modelling Symposium Berlin 2009 (pp. 331-9). UdK Berlin.

[104] Umetani, N., Igarashi, T., \& Mitra, N. J. (2012). Guided exploration of physically valid shapes for furniture design. ACM Tran. Graphics, 31(4), \#86,1-11.

[105] Van Mele, T., \& Block, P. (2011). A novel form finding method for fabric formwork for concrete shells. J. Int. Assoc. Shell Spatial Struct., 52, 217-224

[106] Van Mele, T., Panozzo, D., Sorkine-Hornung, O., \& Block, P. (2014). Best-fit thrust network analysis: Rationalization of freeform meshes. In S. Adriaenssens et al. (Eds.), Shell Structures for Architecture - Form Finding and Optimization (pp. 157-70). Routledge.

[107] Vaxman, A. (2012). Modeling polyhedral meshes with affine maps. Comput. Graph. Forum, 31, 1647-1656. Proc. SGP.

[108] Vaxman, A. (2014). A projective framework for polyhedral mesh modelling. Comput. Graph. Forum, 33

[109] Vouga, E., Höbinger, M., Wallner, J., \& Pottmann, H. (2012). Design of self-supporting surfaces. ACM Trans. Graph., 31(4), \#87,1-11. Proc. SIGGRAPH

[110] Wallner, J. (2012). On the semidiscrete differential geometry of Asurfaces and K-surfaces. J. Geometry, 103, 161-176.

[111] Wang, J., Jiang, C., Bompas, P., Wallner, J., \& Pottmann, H. (2013). Discrete line congruences for shading and lighting. Comput. Graph. Forum, 32(5), 53-62. Proc. SGP.

[112] Whiting, E., Ochsendorf, J., \& Durand, F. (2009). Procedural modeling of structurally-sound masonry buildings. ACM Trans. Graph., 28(5), \#112,1-9. Proc. SIGGRAPH Asia.

[113] Wu, J., \& Kobbelt, L. (2005). Structure recovery via hybrid variational surface approximation. Comput. Graph. Forum, 24(3), 277-284. Proc. Eurographics.

[114] Yan, D.-M., Liu, Y., \& Wang, W. (2006). Quadric surface extraction by 
variational shape approximation. In Geometric Modeling and Processing - GMP 2006 (pp. 73-86). Springer.

[115] Yang, Y.-L., Yang, Y., Pottmann, H., \& Mitra, N. (2011). Shape space exploration of constrained meshes. ACM Trans. Graph., 30(6), \#124,111. Proc. SIGGRAPH Asia.

[116] Zadravec, M., Schiftner, A., \& Wallner, J. (2010). Designing quaddominant meshes with planar faces. Comput. Graph. Forum, 29(5), 1671-1679. Proc. SGP.

[117] Zimmer, H., Campen, M., Bommes, D., \& Kobbelt, L. (2012). Rationalization of triangle-based point-folding structures. Comput. Graph. Forum, 31, 611-620. Proc. Eurographics.

[118] Zimmer, H., Campen, M., Herkrath, R., \& Kobbelt, L. (2012). Variational tangent plane intersection for planar polygonal meshing. In L. Hesselgren et al. (Eds.), Advances in Architectural Geometry 2012 (pp. 319-32). Springer.

[119] Zimmer, H., \& Kobbelt, L. (2014). Zometool rationalization of freeform surfaces. IEEE Trans. Vis. Comput. Graph., 20, 1461-1473.

[120] Zimmer, H., Lafarge, F., Alliez, P., \& Kobbelt, L. (2014). Zometool shape approximation. Graphical Models, 76(5), 390-401. Proc. GMP.

[121] Li, Y., Liu, Y., \& Wang, W. (2014). Planar hexagonal meshing for architecture. IEEE Trans. Vis. Comp. Graphics, 21, 95-106.

[122] Wang, W., Liu, Y., Yan, D.-M., Chan, B., Ling, R., \& Sun, F. (2008) Hexagonal meshes with planar faces. Technical Report TR-2008-13 (CS series) University of Hong Kong. 